\title{
Spin-polarized current and shot noise in the presence of spin flip in a quantum dot via nonequilibrium Green's functions
}

De Souza, Fabricio; Jauho, Antti-Pekka; Egues, J.C.

Published in:

Physical Review B Condensed Matter

Link to article, DOI:

10.1103/PhysRevB.78.155303

Publication date:

2008

Document Version

Publisher's PDF, also known as Version of record

Link back to DTU Orbit

Citation (APA):

De Souza, F., Jauho, A-P., \& Egues, J. C. (2008). Spin-polarized current and shot noise in the presence of spin flip in a quantum dot via nonequilibrium Green's functions. Physical Review B Condensed Matter, 78(15), 155303. https://doi.org/10.1103/PhysRevB.78.155303

\section{General rights}

Copyright and moral rights for the publications made accessible in the public portal are retained by the authors and/or other copyright owners and it is a condition of accessing publications that users recognise and abide by the legal requirements associated with these rights.

- Users may download and print one copy of any publication from the public portal for the purpose of private study or research.

- You may not further distribute the material or use it for any profit-making activity or commercial gain

- You may freely distribute the URL identifying the publication in the public portal 


\title{
Spin-polarized current and shot noise in the presence of spin flip in a quantum dot via nonequilibrium Green's functions
}

\author{
F. M. Souza, ${ }^{1,2,4}$ A. P. Jauho, ${ }^{2,3}$ and J. C. Egues ${ }^{4}$ \\ ${ }^{1}$ International Center for Condensed Matter Physics, Universidade de Brasília, 70904-910, Brasília, DF, Brazil \\ ${ }^{2}$ Department of Micro and Nanotechnology, Technical University of Denmark, DTU Nanotech, Building 345 East, \\ DK-2800, Kongens Lyngby, Denmark \\ ${ }^{3}$ Laboratory of Physics, Helsinki University of Technology, P.O. Box 1100, Helsinki FI-02015 HUT, Finland \\ ${ }^{4}$ Departamento de Física e Informática, Instituto de Física de São Carlos, Universidade de São Paulo, 13560-970 São Carlos, SP, Brazil
}

(Received 7 February 2008; revised manuscript received 13 July 2008; published 3 October 2008)

\begin{abstract}
Using nonequilibrium Green's functions we calculate the spin-polarized current and shot noise in a ferromagnet-quantum-dot-ferromagnet system. Both parallel (P) and antiparallel (AP) magnetic configurations are considered. Coulomb interaction and coherent spin flip (similar to a transverse magnetic field) are taken into account within the dot. We find that the interplay between Coulomb interaction and spin accumulation in the dot can result in a bias-dependent current polarization $\wp$. In particular, $\wp$ can be suppressed in the P alignment and enhanced in the AP case depending on the bias voltage. The coherent spin flip can also result in a switch of the current polarization from the emitter to the collector lead. Interestingly, for a particular set of parameters it is possible to have a polarized current in the collector and an unpolarized current in the emitter lead. We also found a suppression of the Fano factor to values well below 0.5 .
\end{abstract}

DOI: 10.1103/PhysRevB.78.155303

PACS number(s): 72.25. - b, 72.10.Bg, 85.75.-d, 73.63.Kv

\section{INTRODUCTION}

Spin-dependent transport in quantum dots (QDs) is a subject of intense study nowadays due to its relevance to proposed spintronic devices that encompass, for instance, the Datta-Das transistor, ${ }^{1}$ memory devices, ${ }^{2,3}$ and as an ultimate goal, quantum computers. ${ }^{4}$ In particular, the recent progress in the coherent control of electron spins in quantum dots ${ }^{5-7}$ has stimulated even further the research in this field for possible applications in quantum computation and quantum information processing. ${ }^{8}$ In addition to these fascinating technological applications, quantum dots constitute a unique well-controllable system to study fundamental physical aspects of transport in the strong Coulomb-correlated regime and its interplay with spin-dependent effects.

A common geometry used for transport studies in quantum dots consists of two leads weakly coupled to a QD via tunneling barriers. Spin-dependent effects such as spin accumulation and spin-polarized transport can occur in these systems when both leads are (or at least one of them is) ferromagnetic (FM). The junction ferromagnet-quantum-dotferromagnet (FM-QD-FM) resembles the standard tunnel magnetoresistance (TMR) ${ }^{9,10}$ and giant magnetoresistance (GMR) (Ref. 11) geometries composed of an insulator layer sandwiched by two ferromagnetic metallic leads, except for the quantum dot replacing the insulator layer. This system (dot coupled to FM leads) was recently experimentally realized in the context of semiconductor quantum dots ${ }^{12,13}$ and molecules. ${ }^{14-16}$ A wealth of spin-dependent effects has been observed in this system due to the interplay of quantum confinement, Coulomb correlations, Pauli principle, and leadpolarization alignments. For instance, effects such as spin accumulation, ${ }^{17,18}$ spin diode, ${ }^{19,20}$ spin blockade, ${ }^{21-24}$ spin current ringing, 25,26 negative differential conductance, and negative $\mathrm{TMR}^{17,21}$ arise in this context. In order to obtain additional information, not contained in the average current, shot noise has also been analyzed in several spintronic systems. A few examples include shot noise in spin-valve junctions ${ }^{27-30}$ and quantum dots attached to ferromagnetic leads. ${ }^{31-33}$

Here we apply the Keldysh nonequilibrium technique to study spin-polarized transport (current and shot noise) in a FM-QD-FM system (Fig. 1). Both parallel (P) and antiparallel (AP) lead magnetization alignments are considered. The left and the right lead materials are taken to be different, thus resulting in additional effects, not seen for leads with the same material. We analyze both the current and the shot noise in the presence of Coulomb interaction and spin flip in the dot. We find an interplay between spin accumulation and Coulomb interaction that gives rise to a bias-dependent cur-

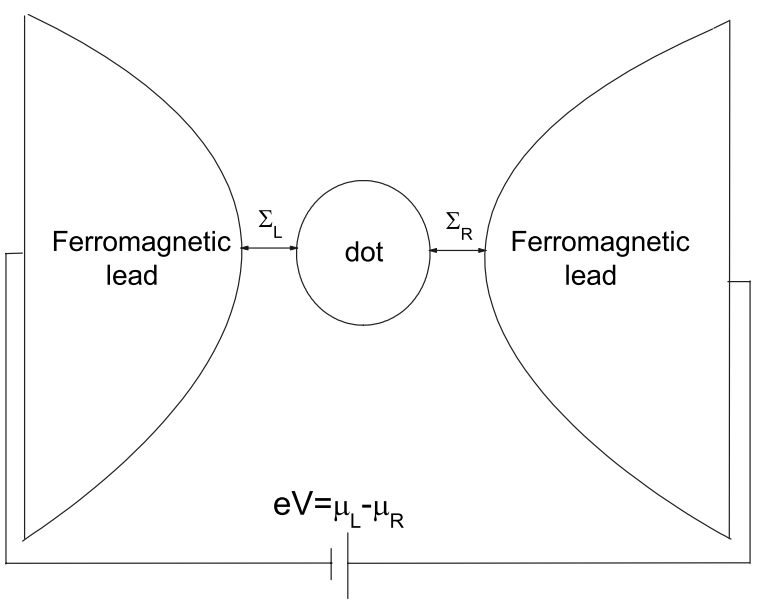

FIG. 1. Schematic of the system studied: a quantum dot coupled to two ferromagnetic leads. On the forward bias the electrons tunnel from the left lead to the right lead via the quantum dot. We consider configurations in which the FM leads are P or AP. The dot has a single orbital level and can hold at most two electrons of opposite spins. Intradot Coulomb interaction and spin flip are considered. 
rent polarization $\wp$. More specifically, $\wp$ can be suppressed or enhanced and have its sign changed depending on the magnetic alignment and the bias voltage. We also note that the spin flip can switch the current polarization as it flows from the emitter to the collector lead. In particular, it is possible to have an unpolarized emitter current and a polarized collector current. For the shot noise, we find that spin flip can suppress it (in the AP case) with Fano factors reaching well below 1/2.

The outline of our paper is as follows: In Sec. II we describe in detail our model Hamiltonian. In Sec. III we present the current and the noise calculations, respectively, including general formulas for these quantities. In Sec. IV we present and discuss numerical results for the current and the shot noise. We summarize our conclusions in Sec. V. Technical details of our calculation are described in the Appendixes A-D.

\section{MODEL SYSTEM AND HAMILTONIAN}

Our system consists of a quantum dot with one quantized level coupled to two ferromagnetic leads via tunneling barriers. While the electrons in the leads are noninteracting, the electrons in the dot experience Coulomb repulsion and spinflip scattering. The system Hamiltonian is

$$
H=H_{L}+H_{R}+H_{D}+H_{T} .
$$

The first three terms in Eq. (1) correspond to the three different regions: left lead, right lead, and dot. The last term $H_{T}$ hybridizes these three regions thus allowing electrons to tunnel from one region to the other. This term gives rise to current in the presence of a bias voltage.

More explicitly, we have for the ferromagnetic leads

$$
H_{\eta}=\sum_{k \sigma} \epsilon_{k \sigma \eta} c_{k \sigma \eta}^{\dagger} c_{k \sigma \eta},
$$

where $\epsilon_{k \sigma \eta}=\epsilon_{k \eta}+(-1)^{\delta_{\sigma \downarrow} \Delta}$ (Stoner model) is the spindependent energy of the electron in lead $\eta=(L, R)$, with the band spin splitting $\Delta, \sigma=\uparrow, \downarrow$ and $\delta_{\uparrow \uparrow(\downarrow \downarrow)}=1 ; \delta_{\downarrow \uparrow(\uparrow \downarrow)}=0$. The operator $c_{k \sigma \eta}\left(c_{k \sigma \eta}^{\dagger}\right)$ destroys (creates) an electron with wave vector $k$ and spin $\sigma$ in lead $\eta$. The dot Hamiltonian is

$$
H_{D}=\sum_{\sigma} \epsilon_{\sigma} d_{\sigma}^{\dagger} d_{\sigma}+U n_{\uparrow} n_{\downarrow}+R\left(d_{\uparrow}^{\dagger} d_{\downarrow}+d_{\downarrow}^{\dagger} d_{\uparrow}\right),
$$

where $\epsilon_{\sigma}$ is the dot level and $d_{\sigma}\left(d_{\sigma}^{\dagger}\right)$ annihilates (creates) an electron in the dot with spin $\sigma$. Our model assumes a single spin-degenerate orbital level in the dot, $\epsilon_{\uparrow}=\epsilon_{\downarrow}=\epsilon_{d}$. More specifically, our dot can be singly occupied by an electron with spin up, down, or doubly occupied by two electrons with opposite spins. We account for the Coulomb interaction in the dot via the Hubbard term with correlation parameter $U$. We assume a linear voltage drop across the system: $\epsilon_{d}=\epsilon_{0}$ $-\frac{e V}{2}$, where $e>0, V$ is the applied voltage, and $\epsilon_{0}$ is the dot level for $V=0$. The left $\mu_{L}$ and the right $\mu_{R}$ chemical potentials are related by $\mu_{L}-\mu_{R}=e V$. Here we assume that $\mu_{L}$ is constant and defines the origin of the energy. For positive bias $\left(\mu_{L}>\mu_{R}\right)$ the left lead is the electron emitter and the right lead is the collector. The last term in Eq. (3) accounts for a coherent spin flip in the dot. ${ }^{34}$ This term can represent, e.g., a local transverse magnetic field that coherently rotates the electron spin, which can be experimentally realized via electron spin resonance (ESR) techniques ${ }^{35}$ or by the Hanle effect. $^{36,37}$

Instead of carrying out the calculations with Hamiltonian (3), we perform the following canonical transformation:

$$
d_{\sigma}=\frac{1}{\sqrt{2}} \sum_{i=1,2}(-1)^{i \delta_{\sigma \downarrow}} d_{i} .
$$

With Eq. (4), the dot Hamiltonian becomes

$$
H_{D}=\sum_{i=1,2}\left[\epsilon_{d}+(-1)^{i} R\right] d_{i}^{\dagger} d_{i}+U n_{1} n_{2}
$$

where $n_{i}=d_{i}^{\dagger} d_{i}$. Note that in Eq. (5) the dot level is split into two levels: $\epsilon_{1}=\epsilon_{d}-R$ and $\epsilon_{2}=\epsilon_{d}+R$. We note that this canonical transformation rotates the spin-quantization axis (e.g., to the direction of a local transverse magnetic field), thus replacing the spin-flip term by a diagonal term with a split level.

The tunneling Hamiltonian in Eq. (1) is

$$
H_{T}=\sum_{k \sigma \eta}\left(t_{k \sigma} c_{k \sigma \eta}^{\dagger} d_{\sigma}+t_{k \sigma}^{*} d_{\sigma}^{\dagger} c_{k \sigma \eta}\right)
$$

where the matrix element $t_{k \sigma}$ connects an electronic state in lead $\eta$ to one in the dot. Observe that the hopping process between the leads and the dot is spin conserving, i.e., $t_{k \sigma}$ does not mix different spin components. Applying the transformation Eq. (4) into Eq. (6) we find

$$
H_{T}=\sum_{k \sigma \eta i} \frac{(-1)^{i \delta_{\sigma \downarrow}}}{\sqrt{2}}\left\{t_{k \sigma}^{*} d_{i}^{\dagger} c_{k \sigma \eta}+t_{k \eta \sigma} c_{k \sigma \eta}^{\dagger} d_{i}\right\} .
$$

Next we calculate current and noise for the model described above.

\section{CURRENT AND NOISE}

The current is calculated in a standard way from the definition $I_{L}(t)=\left\langle\hat{I}_{L}(t)\right\rangle$, where $\hat{I}_{L}(t)=-e \dot{N}_{L}$ is the current operator, with $N_{L}=\sum_{k \sigma} c_{k \sigma L}^{\dagger} c_{k \sigma L}$ being the total number operator, and $\langle\ldots\rangle$ is a thermodynamic average. From the Heisenberg equation $\dot{N}_{L}=i\left[H, N_{L}\right]$, we find

$$
\hat{I}_{L}(t)=i e \sum_{k \sigma}\left[t_{k \sigma} c_{k \sigma \eta}^{\dagger}(t) d_{\sigma}(t)-t_{k \sigma}^{*} d_{\sigma}^{\dagger}(t) c_{k \sigma \eta}(t)\right],
$$

which results in the following current expression: ${ }^{38,39}$

$$
I_{L}(t)=2 e \operatorname{Re} \sum_{k \sigma} t_{k \sigma} i\left\langle c_{k L \sigma}^{\dagger}(t) d_{\sigma}(t)\right\rangle .
$$

A similar expression holds for the right lead current $I_{R}$ $=-e\left\langle\dot{N}_{R}\right\rangle$. Since we are in a stationary regime, we have simply $I_{L}=-I_{R}$. Using the canonical transformation in Eq. (9), we obtain 


$$
I_{L}=2 e \operatorname{Re} \sum_{k \sigma i} t_{k \sigma} \frac{(-1)^{i \delta_{\sigma \downarrow}}}{\sqrt{2}} G_{i k \sigma L}^{<}(t, t),
$$

where $G_{i k \sigma L}^{<}(t, t)=i\left\langle c_{k L \sigma}^{\dagger}(t) d_{i}(t)\right\rangle$ is the lesser Green's function, which is calculated via the Keldysh nonequilibrium technique. ${ }^{40,41}$

As a starting point we construct the complex time Green's function $G_{i k \sigma L}\left(\tau, \tau^{\prime}\right)=-i\left\langle T_{c} d_{i}(\tau) c_{k \sigma L}^{\dagger}\left(\tau^{\prime}\right)\right\rangle$, where $T_{c}$ is the contour time-ordering operator and $\tau$ and $\tau^{\prime}$ are the complex times running along a complex contour. ${ }^{40,41}$ Then we go from the Heisenberg to the interaction picture by introducing the $S$-matrix operator $S=e^{-i \int_{c} d \tau \tilde{H}_{T}(\tau)}$. Here the tilde means that $H_{T}$ is in the interaction picture. After expanding $S$ we find $^{39}$

$$
G_{i k \sigma L}\left(\tau, \tau^{\prime}\right)=t_{k \sigma}^{*} \sum_{j} \frac{(-1)^{j \delta_{\sigma \downarrow}}}{\sqrt{2}} \int_{c} d \tau_{1} G_{i j}\left(\tau, \tau_{1}\right) g_{k \sigma L}\left(\tau_{1}, \tau^{\prime}\right),
$$

where $\quad G_{i j}\left(\tau, \tau_{1}\right)=-i\left\langle T_{c} d_{i}(\tau) d_{j}^{\dagger}\left(\tau_{1}\right)\right\rangle \quad$ and $\quad g_{k \sigma L}\left(\tau_{1}, \tau^{\prime}\right)$ $=-i\left\langle T_{c} \widetilde{c}_{k \sigma L}\left(\tau_{1}\right) \tilde{c}_{k \sigma L}^{\dagger}\left(\tau^{\prime}\right)\right\rangle$. Note that while $G_{i j}\left(\tau, \tau_{1}\right)$ is in the Heisenberg picture, $g_{k \sigma L}\left(\tau_{1}, \tau^{\prime}\right)$ is in the interaction picture (denoted by the tilde operators). This "separability" of the interaction and Heisenberg pictures follows from the assumption of noninteracting electrons in the leads. This allows us to put the "difficult" part of the analysis entirely in the dot Green's functions, which contain the Coulomb interaction, the spin flip, and the coupling to leads.

The next step is to apply Langreth's analytical continuation rules $^{41}$ to Eq. (11), to find the lesser Green's function appearing in Eq. (10). This yields

$$
\begin{aligned}
G_{i k \sigma L}^{<}\left(t, t^{\prime}\right)= & t_{k L \sigma}^{*} \sum_{j} \frac{(-1)^{j \delta_{\sigma \downarrow}}}{\sqrt{2}} \int d t_{1}\left\{G_{i j}^{r}\left(t, t_{1}\right) g_{k \sigma L}^{<}\left(t_{1}, t^{\prime}\right)\right. \\
& \left.+G_{i j}^{<}\left(t, t_{1}\right) g_{k \sigma L}^{a}\left(t_{1}, t^{\prime}\right)\right\},
\end{aligned}
$$

where the labels $r, a$, and $<$ mean retarded, greater, and lesser, respectively. The calculation of the retarded $G_{i j}^{r}$ and lesser $G_{i j}^{<}$dot Green's functions is presented in Appendix A.

Using this result in Eq. (10) we arrive at

$$
I_{L}=2 e \operatorname{Re} \sum_{i j} \int d t_{1}\left\{G_{i j}^{r}\left(t, t_{1}\right) \Sigma_{j i}^{<}\left(t_{1}, t\right)+G_{i j}^{<}\left(t, t_{1}\right) \Sigma_{j i}^{a}\left(t_{1}, t\right)\right\},
$$

where $\Sigma_{j i}^{L(<, a)}\left(t_{1}, t\right)=\frac{1}{2} \sum_{k \sigma}\left|t_{k \sigma}\right|^{2}(-1)^{(i+j)} \delta_{\sigma \downarrow} g_{k \sigma L}^{(<, a)}\left(t_{1}, t\right)$, with the lesser Green's function $g_{k \sigma L}^{<}\left(t_{1}, t\right)=i\left\langle\widetilde{c}_{k \sigma L}^{\dagger}(t) \widetilde{c}_{k \sigma L}\left(t_{1}\right)\right\rangle$ and the advanced one $g_{k \sigma L}^{a}\left(t_{1}, t\right)=i \theta\left(t-t_{1}\right)\left\langle\left\{\tilde{c}_{k \sigma L}\left(t_{1}\right), \widetilde{c}_{k \sigma L}^{\dagger}(t)\right\}\right\rangle$; here the curly brackets denote an anticommutator.

In the steady-state regime the Fourier transforms of the Green's functions result in single frequency Green's functions. Since this is the regime of interest here, we state for later use the Fourier transform of the leads Green's functions,

$$
g_{k \sigma \eta}^{a}(\epsilon)=g_{k \sigma \eta}^{r^{*}}(\epsilon)=\frac{1}{\epsilon-\epsilon_{k \sigma \eta}-i \delta},
$$

$$
g_{k \sigma \eta}^{<}(\epsilon)=2 \pi i \eta_{\eta}(\epsilon) \delta\left(\epsilon-\epsilon_{k \sigma \eta}\right),
$$

where $n_{\eta}$ is the Fermi distribution function of lead $\eta$.

\section{A. Average current in the stationary regime}

In a stationary regime all of the Green's functions depend on only $t-t_{1}$, yielding the Fourier transform

$$
\begin{aligned}
I_{L} & =2 e \operatorname{Re} \int \frac{d \epsilon}{2 \pi} \sum_{i j}\left\{G_{i j}^{r}(\epsilon) \Sigma_{j i}^{L<}(\epsilon)+G_{i j}^{<}(\epsilon) \Sigma_{j i}^{L a}(\epsilon)\right\} \\
& =i e \int \frac{d \boldsymbol{\epsilon}}{2 \pi} \operatorname{Tr}\left\{\boldsymbol{\Gamma}^{L}\left[\left(\mathbf{G}^{r}-\mathbf{G}^{a}\right) n_{L}+\mathbf{G}^{<}\right]\right\},
\end{aligned}
$$

with

$$
\Gamma^{L}=\frac{1}{2}\left(\begin{array}{cc}
\Gamma_{\uparrow}^{L}+\Gamma_{\downarrow}^{L} & \Gamma_{\uparrow}^{L}-\Gamma_{\downarrow}^{L} \\
\Gamma_{\uparrow}^{L}-\Gamma_{\downarrow}^{L} & \Gamma_{\uparrow}^{L}+\Gamma_{\downarrow}^{L}
\end{array}\right),
$$

where $\Gamma_{\sigma}^{L}=2 \pi \Sigma_{k}\left|t_{k \sigma}\right|^{2} \delta\left(\epsilon-\epsilon_{k \sigma L}\right)$ is the linewidth function. In what follows we neglect the energy dependence of $\Gamma_{\sigma}^{\eta}$ (wideband limit), which will be taken as a constant phenomenological parameter.

\section{B. Spin-resolved currents}

From Eqs. (16) and (17) we can also determine the spinresolved components of the average current,

$$
\begin{aligned}
I_{L}^{\sigma}= & i e \int \frac{d \epsilon}{2 \pi} \operatorname{Tr}\left\{\frac{\Gamma_{\sigma}^{L}}{2}\left(\begin{array}{cc}
1 & (-1)^{\delta_{\sigma \downarrow}} \\
(-1)^{\delta_{\sigma \downarrow}} & 1
\end{array}\right)\right. \\
& \left.\times\left[n_{L}\left(\mathbf{G}^{r}-\mathbf{G}^{a}\right)+\mathbf{G}^{<}\right]\right\} .
\end{aligned}
$$

A similar result holds for $I_{R}^{\sigma}$. Equation (18) gives the spinpolarized current components with their polarization axes defined along the magnetic moment of the leads. In the present study no spin torque is considered, which makes the projected current a relevant quantity to investigate. In the presence of spin torque more general definitions for spinresolved charge currents and spin currents should be used. A general expression for the spin current in the presence of spin transfer was recently derived in Ref. 42.

\section{Noise definition}

Fluctuations of the current are interesting because they can give additional information about the system beyond that provided by the average current alone. ${ }^{43}$ Here we derive an expression for the current fluctuations, which include both thermal and shot noises. The thermal noise is related to fluctuations in the occupations of the leads due to thermal excitation, and it vanishes at zero temperature. Shot noise is an unavoidable temporal fluctuation of the current due to the granularity of the electron charge. It is nonzero only for finite bias, i.e., it is a nonequilibrium property. In the linearresponse regime the fluctuation-dissipation theorem holds, yielding the relation $S(\omega)=4 k_{B} T G(\omega)$, where $G(\omega)$ is the conductance. ${ }^{43}$ Hence, in equilibrium the noise contains the 
same information as the conductance. Away from equilibrium this relation is no longer valid and the noise spectrum can provide additional information.

We define noise via $S_{\eta \eta^{\prime}}=\left\langle\left\{\delta \hat{I}_{\eta}(t), \delta \hat{I}_{\eta^{\prime}}\left(t^{\prime}\right)\right\}\right\rangle$, where $\delta \hat{I}_{\eta}(t)=\hat{I}_{\eta}(t)-I_{\eta}$ is the current fluctuation at a time $t$ in lead $\eta$. Equivalently,

$$
S_{\eta \eta^{\prime}}\left(t, t^{\prime}\right)=\left\langle\left\{\hat{I}_{\eta}(t), \hat{I}_{\eta^{\prime}}\left(t^{\prime}\right)\right\}\right\rangle-2 I_{\eta}^{2}
$$

where we use the fact that $I_{\eta}=\left\langle\hat{I}_{\eta}(t)\right\rangle=\left\langle\hat{I}_{\eta^{\prime}}\left(t^{\prime}\right)\right\rangle$ in the stationary regime. Using the current operator $\hat{I}_{\eta}[$ Eq. (8)] and Eq. (4) into Eq. (19), we obtain

$$
\begin{aligned}
S_{\eta \eta^{\prime}}\left(t, t^{\prime}\right)= & (i e)^{2} \sum_{k k^{\prime} \sigma \sigma^{\prime} i j} \frac{1}{2}(-1)^{i \delta_{\sigma \downarrow}(-1)^{j \delta_{\sigma^{\prime} \downarrow}}} \\
& \times\left\{t_{k \sigma} t_{k^{\prime} \sigma^{\prime}}\left\langle c_{k \sigma \eta}^{\dagger}(t) d_{i}(t) c_{k^{\prime} \sigma^{\prime} \eta^{\prime}}^{\dagger}\left(t^{\prime}\right) d_{j}\left(t^{\prime}\right)\right\rangle\right. \\
& -t_{k \sigma} t_{k^{\prime} \sigma^{\prime}}^{*}\left\langle c_{k \sigma \eta}^{\dagger}(t) d_{i}(t) d_{j}^{\dagger}\left(t^{\prime}\right) c_{k^{\prime} \sigma^{\prime} \eta^{\prime}}\left(t^{\prime}\right)\right\rangle \\
& -t_{k \sigma}^{*} t_{k^{\prime} \sigma^{\prime}}\left\langle d_{i}^{\dagger}(t) c_{k \sigma \eta}(t) c_{k^{\prime} \sigma^{\prime} \eta^{\prime}}^{\dagger}\left(t^{\prime}\right) d_{j}\left(t^{\prime}\right)\right\rangle \\
& \left.+t_{k \sigma}^{*} t_{k^{\prime} \sigma^{\prime}}^{*}\left\langle d_{i}^{\dagger}(t) c_{k \sigma \eta}(t) d_{j}^{\dagger}\left(t^{\prime}\right) c_{k^{\prime} \sigma^{\prime} \eta^{\prime}}\left(t^{\prime}\right)\right\rangle\right\} \\
& + \text { H.c. }-2 I_{\eta}^{2} .
\end{aligned}
$$

\section{Noise in terms of Green's functions}

Each $\langle\ldots\rangle$ term in Eq. (20) can be expressed in terms of a Green's function. Defining the two-particle Green's functions,

$$
\begin{aligned}
& g_{1}^{(2)}\left(\tau, \tau^{\prime}\right)=i^{2}\left\langle T_{c} c_{k \sigma \eta}^{\dagger}(\tau) d_{i}(\tau) c_{k^{\prime} \sigma^{\prime} \eta^{\prime}}^{\dagger}\left(\tau^{\prime}\right) d_{j}\left(\tau^{\prime}\right)\right\rangle, \\
& g_{2}^{(2)}\left(\tau, \tau^{\prime}\right)=i^{2}\left\langle T_{c} c_{k \sigma \eta}^{\dagger}(\tau) d_{i}(\tau) d_{j}^{\dagger}\left(\tau^{\prime}\right) c_{k^{\prime} \sigma^{\prime} \eta^{\prime}}\left(\tau^{\prime}\right)\right\rangle, \\
& g_{3}^{(2)}\left(\tau, \tau^{\prime}\right)=i^{2}\left\langle T_{c} d_{i}^{\dagger}(\tau) c_{k \sigma \eta}(\tau) c_{k^{\prime} \sigma^{\prime} \eta^{\prime}}^{\dagger}\left(\tau^{\prime}\right) d_{j}\left(\tau^{\prime}\right)\right\rangle, \\
& g_{4}^{(2)}\left(\tau, \tau^{\prime}\right)=i^{2}\left\langle T_{c} d_{i}^{\dagger}(\tau) c_{k \sigma \eta}(\tau) d_{j}^{\dagger}\left(\tau^{\prime}\right) c_{k^{\prime} \sigma^{\prime} \eta^{\prime}}\left(\tau^{\prime}\right)\right\rangle,
\end{aligned}
$$

we can write Eq. (20) as

$$
\begin{aligned}
& S_{\eta \eta^{\prime}}\left(t, t^{\prime}\right)=e^{2} \sum_{k k^{\prime} \sigma \sigma^{\prime} i j} \frac{1}{2}(-1)^{i \delta_{\sigma \downarrow}}(-1)^{j \delta_{\sigma^{\prime} \downarrow} \downarrow\left\{t_{k \sigma} t_{k^{\prime} \sigma^{\prime}} g_{1}^{(2)>}\right.}\left(t, t^{\prime}\right) \\
& -t_{k \sigma} t_{k^{\prime} \sigma^{\prime}}^{*} g_{2}^{(2)>}\left(t, t^{\prime}\right)-t_{k \sigma}^{*} t_{k^{\prime} \sigma^{\prime}} g_{3}^{(2)>}\left(t, t^{\prime}\right) \\
& \left.+t_{k \sigma}^{*} t_{k^{\prime} \sigma^{\prime}}^{*} g_{4}^{(2)>}\left(t, t^{\prime}\right)\right\}+ \text { H.c. }-2 I_{\eta}^{2},
\end{aligned}
$$

where $g_{i}^{(2)>}\left(t, t^{\prime}\right)$ is obtained from the complex time Green's function $g_{i}^{(2)}\left(\tau, \tau^{\prime}\right)$ via analytical continuation. Similarly to the current calculation where we develop an $S$-matrix expansion in $G_{\sigma k \sigma L}\left(\tau, \tau^{\prime}\right)$ to obtain $G_{\sigma k \sigma L}^{<}\left(t, t^{\prime}\right)$, here we expand the $S$ matrix in $g_{i}^{(2)}\left(\tau, \tau^{\prime}\right)$ and then obtain $g_{i}^{(2)>}\left(t, t^{\prime}\right)$. This procedure follows the standard calculations proposed in Ref. 39 to derive the current equation. The details of this $S$-matrix expansion are presented in Appendix B; here we simply state the results,

$$
\begin{aligned}
g_{1}^{(2)}\left(\tau, \tau^{\prime}\right)= & \frac{1}{2} \sum_{i_{1}, i_{2}=1,2}(-1)^{i_{1} \delta_{\sigma \downarrow}(-1)^{i_{2} \delta_{\sigma^{\prime}}}} \\
& \times t_{k \sigma^{*}}^{*} t_{k^{\prime} \sigma^{\prime}}^{*} \iint d \tau_{1} d \tau_{2} g_{k \sigma \eta}\left(\tau_{1}, \tau\right) g_{k^{\prime} \sigma^{\prime} \eta^{\prime}}\left(\tau_{2}, \tau^{\prime}\right) \\
& \times\left\{G_{i i_{1}}\left(\tau, \tau_{1}\right) G_{j i_{2}}\left(\tau^{\prime}, \tau_{2}\right)-G_{i i_{2}}\left(\tau, \tau_{2}\right) G_{j i_{1}}\left(\tau^{\prime}, \tau_{1}\right)\right\}
\end{aligned}
$$

and

$$
\begin{aligned}
g_{2}^{(2)}\left(\tau, \tau^{\prime}\right)= & -\delta_{k \sigma \eta, k^{\prime} \sigma^{\prime} \eta^{\prime}} g_{k \sigma \eta}\left(\tau^{\prime}, \tau\right) G_{i j}\left(\tau, \tau^{\prime}\right) \\
& +\frac{1}{2} \sum_{i_{1}, i_{2}=1,2}(-1)^{i_{1} \delta_{\sigma \downarrow}}(-1)^{i_{2} \delta_{\sigma^{\prime} \downarrow}} \\
& \times t_{k \sigma}^{*} t_{k^{\prime} \sigma^{\prime}} \iint d \tau_{1} d \tau_{2} g_{k \sigma \eta}\left(\tau_{1}, \tau\right) g_{k^{\prime} \sigma^{\prime} \eta^{\prime}}\left(\tau^{\prime}, \tau_{2}\right) \\
& \times\left\{G_{i i_{1}}\left(\tau, \tau_{1}\right) G_{i_{1} j}\left(\tau_{2}, \tau^{\prime}\right)-G_{i j}\left(\tau, \tau^{\prime}\right) G_{i_{2} i_{1}}\left(\tau_{2}, \tau_{1}\right)\right\}
\end{aligned}
$$

Equations (22) and (23) hold on a Hartree-Fock or other mean-field theory (see details in Appendix B). The other two Green's functions $g_{3}^{(2)}$ and $g_{4}^{(2)}$ are given by

$$
g_{3}^{(2)}\left(\tau, \tau^{\prime}\right)=g_{2}^{(2)^{*}}\left(\tau, \tau^{\prime}\right)
$$

and

$$
g_{4}^{(2)}\left(\tau, \tau^{\prime}\right)=g_{1}^{(2)^{*}}\left(\tau, \tau^{\prime}\right) .
$$

From a diagrammatic point of view the terms in Eqs. (22) and (23) involving

$$
G_{i i_{1}}\left(\tau, \tau_{1}\right) g_{k \sigma \eta}\left(\tau_{1}, \tau\right) G_{j i_{2}}\left(\tau^{\prime}, \tau_{2}\right) g_{k^{\prime} \sigma^{\prime} \eta^{\prime}}\left(\tau_{2}, \tau^{\prime}\right)
$$

and

$$
G_{\sigma \sigma}\left(\tau, \tau_{1}\right) g_{k \sigma \eta}\left(\tau_{1}, \tau\right) g_{k^{\prime} \sigma^{\prime} \eta^{\prime}}\left(\tau^{\prime}, \tau_{2}\right) G_{\sigma^{\prime} \sigma^{\prime}}\left(\tau_{2}, \tau^{\prime}\right)
$$

are disconnected. These disconnected terms, together with similar ones in the equations for $g_{3}^{(2)}\left(\tau, \tau^{\prime}\right)$ and $g_{4}^{(2)}\left(\tau, \tau^{\prime}\right)$, cancel identically the term $2 I_{\eta}^{2}$ of Eq. (21) (see Appendix C). So we can say that this corresponds to the linked cluster expansion to the noise. The other terms in Eqs. (22) and (23) give the connected diagrams and thus can give a contribution to the noise. Substituting the connected terms of Eqs. (22)-(25) into Eq. (21), we find

$$
\begin{aligned}
S_{\eta \eta^{\prime}}\left(t, t^{\prime}\right)= & e^{2} \sum_{k \sigma}\left|t_{k \sigma}\right|^{2}\left\{\delta_{\eta, \eta^{\prime}} \sum_{i j}(-1)^{(i+j) \delta_{\sigma \downarrow}}\right. \\
& \times\left[g_{k \sigma \eta}^{>}\left(t, t^{\prime}\right) G_{j i}^{<}\left(t^{\prime}, t\right)+G_{i j}^{>}\left(t, t^{\prime}\right) g_{k \sigma \eta}^{<}\left(t^{\prime}, t\right)\right] \\
& -e^{2} \sum_{k k^{\prime} \sigma \sigma^{\prime}}\left|t_{k \sigma}\right|^{2}\left|t_{k^{\prime} \sigma^{\prime}}\right|^{2} \int_{c} \int_{c} d \tau_{1} d \tau_{2} \\
& \times \frac{1}{4} \sum_{i, j, i_{1}, i_{2}=1,2}(-1)^{\left(i+i_{1}\right) \delta_{\sigma \downarrow}(-1)^{\left(j+i_{2}\right)} \delta_{\sigma^{\prime} \downarrow}} \\
& \times\left\{G_{i i_{2}}\left(t, \tau_{2}\right) g_{k^{\prime} \sigma^{\prime} \eta^{\prime}}\left(\tau_{2}, t^{\prime}\right) G_{j i_{1}}\left(t^{\prime}, \tau_{1}\right) g_{k \sigma \eta}\left(\tau_{1}, t\right)\right. \\
& -G_{i j}\left(t, t^{\prime}\right) g_{k^{\prime} \sigma^{\prime} \eta^{\prime}}\left(t^{\prime}, \tau_{2}\right) G_{i_{2} i_{1}}\left(\tau_{2}, \tau_{1}\right) g_{k \sigma \eta}\left(\tau_{1}, t\right)
\end{aligned}
$$




$$
\begin{aligned}
& -g_{k \sigma \eta}\left(t, \tau_{1}\right) G_{i_{1} i_{2}}\left(\tau_{1}, \tau_{2}\right) g_{k^{\prime} \sigma^{\prime} \eta^{\prime}}\left(\tau_{2}, t^{\prime}\right) G_{j i}\left(t^{\prime}, t\right) \\
& \left.+g_{k \sigma \eta}\left(t, \tau_{1}\right) G_{i_{1} j}\left(\tau_{1}, t^{\prime}\right) g_{k^{\prime} \sigma^{\prime} \eta^{\prime}}\left(t^{\prime}, t_{2}\right) G_{i_{2} i}\left(\tau_{2}, t\right)\right\}^{t>t^{\prime}} \\
& + \text { H.c., }
\end{aligned}
$$

where the superscript $t>t^{\prime}$ means that an analytical continuation should be performed by applying Langreth's rules.

\section{E. Zero-frequency shot noise}

The shot noise is defined as the Fourier transform of $S_{\eta \eta^{\prime}}\left(t-t^{\prime}\right)$, which in the stationary regime reads

$$
S_{\eta \eta^{\prime}}(\omega)=\int_{-\infty}^{\infty} d\left(t-t^{\prime}\right) e^{i \omega\left(t-t^{\prime}\right)} S_{\eta \eta^{\prime}}\left(t-t^{\prime}\right) .
$$

Using the analytical continuation of Eq. (28) into Eq. (29) we find the following zero-frequency shot noise: ${ }^{44}$

$$
\begin{aligned}
S_{L L}(\omega=0)= & \frac{e^{2}}{\pi} \int d \epsilon \operatorname{Tr}\left\{i n_{L} \boldsymbol{\Gamma}^{L} \mathbf{G}^{>}-i\left(1-n_{L}\right) \boldsymbol{\Gamma}^{L} \mathbf{G}^{<}\right. \\
& +\boldsymbol{\Gamma}^{L} \mathbf{G}^{>} \boldsymbol{\Gamma}^{L} \mathbf{G}^{<}+\boldsymbol{\Gamma}^{L}\left(\mathbf{G}^{r}-\mathbf{G}^{a}\right) \boldsymbol{\Gamma}^{L}\left(n_{L} \mathbf{G}^{>}\right. \\
& \left.-\left(1-n_{L}\right) \mathbf{G}^{<}\right)-n_{L}\left(1-n_{L}\right)\left(\mathbf{G}^{a} \boldsymbol{\Gamma}^{L} \mathbf{G}^{a} \boldsymbol{\Gamma}^{L}\right. \\
& \left.\left.+\mathbf{G}^{r} \boldsymbol{\Gamma}^{L} \mathbf{G}^{r} \boldsymbol{\Gamma}^{L}\right)\right\},
\end{aligned}
$$

where $\mathbf{G}^{>}$satisfies the identity $\mathbf{G}^{>}-\mathbf{G}^{<}=\mathbf{G}^{r}-\mathbf{G}^{a}$. All the Green's functions in Eq. (30) are in the frequency domain. In our analysis we take only the component $\eta=\eta^{\prime}=L$. Since the dc noise is independent of position, we have simply $S_{L L}(0)$ $=S_{R R}(0)=-S_{L R}(0)=-S_{R L}(0)$. Equation (30) can be expressed in a standard form as follows ${ }^{45,46}$ (Appendix D):

$$
\begin{aligned}
S_{L L}(\omega=0) & =\frac{e^{2}}{\pi} \int d \epsilon \operatorname{Tr}\left\{\left[n_{L}\left(1-n_{L}\right)+n_{R}\left(1-n_{R}\right)\right] \mathbf{T}(\epsilon)\right. \\
& \left.+\left(n_{L}-n_{R}\right)^{2} \mathbf{T}(\epsilon)[1-\mathbf{T}(\epsilon)]\right\},
\end{aligned}
$$

with the transmission matrix $\mathbf{T}=\boldsymbol{\Gamma}^{L} \mathbf{G}^{r} \boldsymbol{\Gamma}^{R} \mathbf{G}^{a}$. In the calculation leading to Eqs. (30) and (31) we have truncated an $S$-matrix expansion by breaking two-particle Green's function into products of one-particle Green's functions. This procedure holds in a mean-field theory. Thus, for a consistent application of Eqs. (30) and (31), a similar approximation (Hartree-Fock-like) for the Green's functions should be made (see Appendix A). Some limitations imposed by this approximation are discussed in the end of Sec. IV. ${ }^{47}$

\section{F. Model for the FM leads}

The ferromagnetism of the leads is considered via the spin-dependent parameter $\Gamma_{\sigma}^{\eta}$. From the Stoner model, for instance, we can see that the density of states for spin-up electrons of the lead is shifted with respect to that of the spin-down electrons. Since $\Gamma_{\sigma}^{\eta}$ contains information about the spin-dependent density of states, it is expected that $\Gamma_{\uparrow}^{\eta}$ $\neq \Gamma_{\downarrow}^{\eta}{ }^{\eta 48}$ Following Ref. 49 we define $\Gamma_{\sigma}^{L}=\Gamma_{0}\left[1+(-1)^{\delta_{\sigma \downarrow}} p_{L}\right]$. The parameter $\Gamma_{0}$ gives the strength of the lead-to-dot coupling and $p_{L}$ is a parameter describing the degree of spin polarization of the left lead. ${ }^{50}$ Note that $\Gamma_{\uparrow}^{L}>\Gamma_{\downarrow}^{L}$ for $p_{L} \neq 0$. This means that the population for spin up around the Fermi

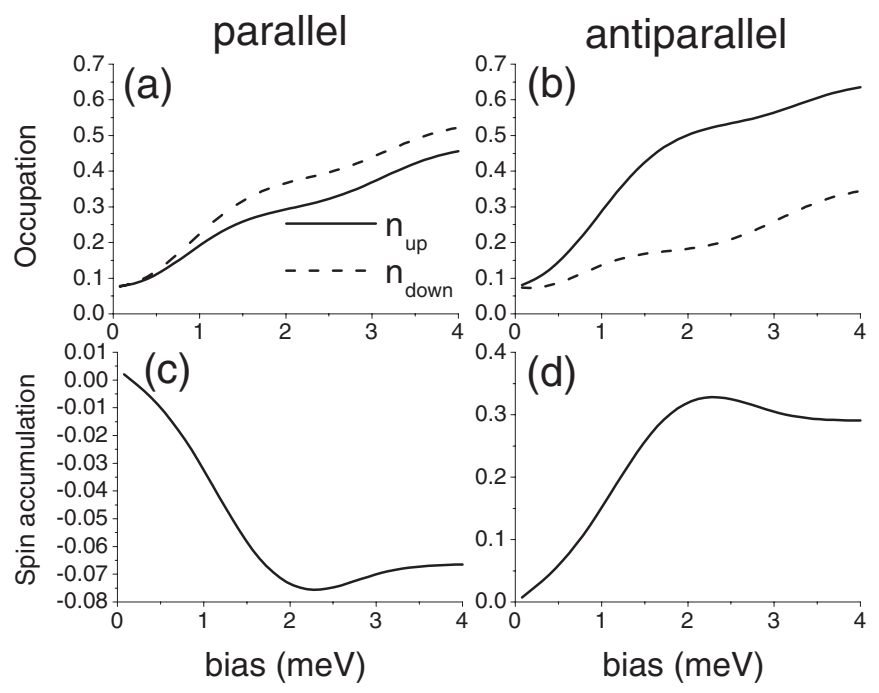

FIG. 2. Spin-resolved occupations $n_{\uparrow}$ and $n_{\downarrow}$ and the spin accumulation, $m=n_{\uparrow}-n_{\downarrow}$, against bias voltage in both parallel and antiparallel FM lead alignments. In the P case $n_{\uparrow}<n_{\downarrow}$, while in the AP alignment $n_{\uparrow}>n_{\downarrow}$. These imbalances give rise to spin-dependent population suppressions in the bias range 1-3 meV. This translates into a tunable $m$ with the bias voltage. Parameters: $p_{L}=0.23, p_{R}$ $=0.35, \quad k_{B} T=200 \mu \mathrm{eV}, \quad \Gamma_{0}=10 \mu \mathrm{eV}, \quad \epsilon_{0}=0.5 \mathrm{meV}, \quad$ and $U$ $=1 \mathrm{meV}$.

energy in the left lead is greater than the population for spin down. Similarly, for the right ferromagnetic lead we assume $\Gamma_{\sigma}^{R}=\Gamma_{0}\left[1+(-1)^{\delta_{\sigma}} p_{R}\right]$ for the $\mathrm{P}$ alignment and $\Gamma_{\sigma}^{R}$ $=\Gamma_{0}\left[1-(-1)^{\delta_{\sigma}} p_{R}\right]$ for the AP alignment. Note that for the $\mathrm{P}$ case we have $\Gamma_{\sigma}^{L}=\Gamma_{\sigma}^{R}$ and for the AP configuration $\Gamma_{\sigma}^{L}=\Gamma_{\bar{\sigma}}^{R}$, with $\bar{\sigma}$ being the opposite of $\sigma$. In the present work we mostly discuss the $p_{L} \neq p_{R}$ case, i.e., a geometry in which the left and right leads are composed of different materials $(\mathrm{Ni}$ and $\mathrm{Co}$, for instance).

\section{G. Numerical procedure}

The numerical results are obtained following a selfconsistent procedure. We calculate the average,

$$
\left\langle\hat{n}_{i j}\right\rangle=\left\langle d_{i}^{\dagger} d_{j}\right\rangle=\int \frac{d \epsilon}{2 \pi i} G_{j i}^{<}(\epsilon),
$$

self-consistently with Eqs. (A9)-(A15). When converged solutions for the expectation values $\left\langle d_{i}^{\dagger} d_{j}\right\rangle$ and dot Green's functions are found, we determine the current [Eq. (18)] and the noise [Eq. (30)]. This iterative schema is performed for each bias voltage.

\section{RESULTS}

\section{A. Spin-resolved electronic occupations}

Occupations. Figures 2(a) and 2(b) show the spin-up and spin-down occupations of the dot for both P and AP alignments. In the $\mathrm{P}$ case the dot has a net spin-down polarization with $n_{\uparrow}<n_{\downarrow}$, while in the AP configuration $n_{\uparrow}>n_{\downarrow}$. These spin imbalances in the dot can be easily understood in terms of the tunneling rates $\Gamma_{\sigma}^{\eta}$ adopted. The parameters $p_{L}=0.23$ 
and $p_{R}=0.35$ used here ${ }^{51}$ yield the following tunneling rates in the parallel case: $\Gamma_{\uparrow}^{L}=12.3 \mu \mathrm{eV}, \quad \Gamma_{\downarrow}^{L}=7.7 \mu \mathrm{eV}, \quad \Gamma_{\uparrow}^{R}$ $=13.5 \mu \mathrm{eV}$, and $\Gamma_{\downarrow}^{R}=6.5 \mu \mathrm{eV}$. In the AP case the values of the tunneling rates to the right lead are swapped $\left(\Gamma_{\uparrow}^{R} \rightleftarrows \Gamma_{\downarrow}^{R}\right)$. From these rates we conclude that in the $\mathrm{P}$ case a spin-up electron leaves the dot faster than it comes in. The opposite happens for a spin-down electron. The imbalance of these in/out tunneling rates results in a larger spin-down occupation in the parallel case, i.e., $n_{\uparrow}<n_{\downarrow}$ [Fig. 2(a)]. By the same token, in the AP alignment we have $n_{\uparrow}>n_{\downarrow}$ as seen in Fig. 2(b).

Spin accumulation. In Figs. 2(c) and 2(d) we show the spin accumulation $\left(m=n_{\uparrow}-n_{\downarrow}\right)$ as a function of the bias voltage. In the zero-bias limit $m$ is essentially zero. When the bias increases, the spin accumulation in the $\mathrm{P}$ case assume negative values. In contrast, in the AP alignment, $m$ is enhanced. In particular, in the bias range corresponding to a singly occupied dot $(1-3 \mathrm{meV})^{52}$ the additional suppression [Fig. 2(c)] or the enhancement [Fig. 2(d)] of $m$ is due to the spin-dependent population suppression that takes place in the presence of Coulomb interaction and spin accumulation. More specifically, in the AP case due to Coulomb interaction $n_{\uparrow}$ tends to suppress more strongly $n_{\downarrow}$ than otherwise. This translates into an enhancement of $m$. In the $\mathrm{P}$ alignment the spin-up occupation $n_{\uparrow}$ is more suppressed than $n_{\downarrow}$, thus $m$ becomes more negative [Fig. 2(c)]. We emphasize that this effect happens for both the P and AP alignments because we assume $p_{L} \neq p_{R}$. For equal leads we find $n_{\uparrow}=n_{\downarrow}$ in the $\mathrm{P}$ case so that $m$ remains zero in this configuration.

\section{B. Current and its polarization}

Current. Figures 3(a) and 3(b) show the current in the P and AP cases for $U=1 \mathrm{meV}$ and $R=0$. Similarly to the occupations, some features of the spin-up and spin-down currents can be understood in terms of the tunneling rates. For instance, their saturation values (second plateau) can be easily calculated from the standard expression, ${ }^{53}$

$$
I_{\eta}^{\sigma}=e \frac{\Gamma_{\sigma}^{L} \Gamma_{\sigma}^{R}}{\Gamma_{\sigma}^{L}+\Gamma_{\sigma}^{R},}
$$

which gives $I_{\eta}^{\uparrow}>I_{\eta}^{\downarrow}$ and $I_{\eta}^{\uparrow}<I_{\eta}^{\downarrow}$ in the P and AP cases, respectively. For the first plateau Eq. (33) is not valid and these inequalities can change. ${ }^{54}$

In the $\mathrm{P}$ case $\left[\right.$ Fig. 3(c)] $I_{\uparrow}$ is more strongly suppressed than $I_{\downarrow}$ due to the interplay of spin accumulation $\left(n_{\uparrow}<n_{\downarrow}\right)$ and Coulomb interaction. This results in a suppression of the current polarization $\left[\wp=\left(I_{\uparrow}-I_{\downarrow}\right) /\left(I_{\uparrow}+I_{\downarrow}\right)\right]$ in the range $1-3$ meV. On the other hand, in the AP case [Fig. 3(d)] $I_{\eta}^{\downarrow}$ is more suppressed than $I_{\eta}^{\uparrow}$ due to the inverted inequality $n_{\uparrow}>n_{\downarrow}$, thus resulting in an enhancement of $\wp$.

Spin-flip effects. In Fig. 4 we show the current polarization against bias voltage for distinct spin-flip parameter $R$. The polarization is calculated for the left and right leads according to the formula $\wp^{\eta}=\left(I_{\eta}^{\uparrow}-I_{\eta}^{\downarrow}\right) /\left(I_{\eta}^{\uparrow}+I_{\eta}^{\downarrow}\right)$, where $\eta$ $=L, R$. For $R=0$ (solid line) we have $\wp^{L}=\wp^{R}$ for all biases. This curve is the same as seen in Fig. 3(d). When $R \neq 0$ these two polarizations depart from each other. The $\wp^{L}$ increases with $R$ tending to reach the left lead-polarization value $p_{L}$

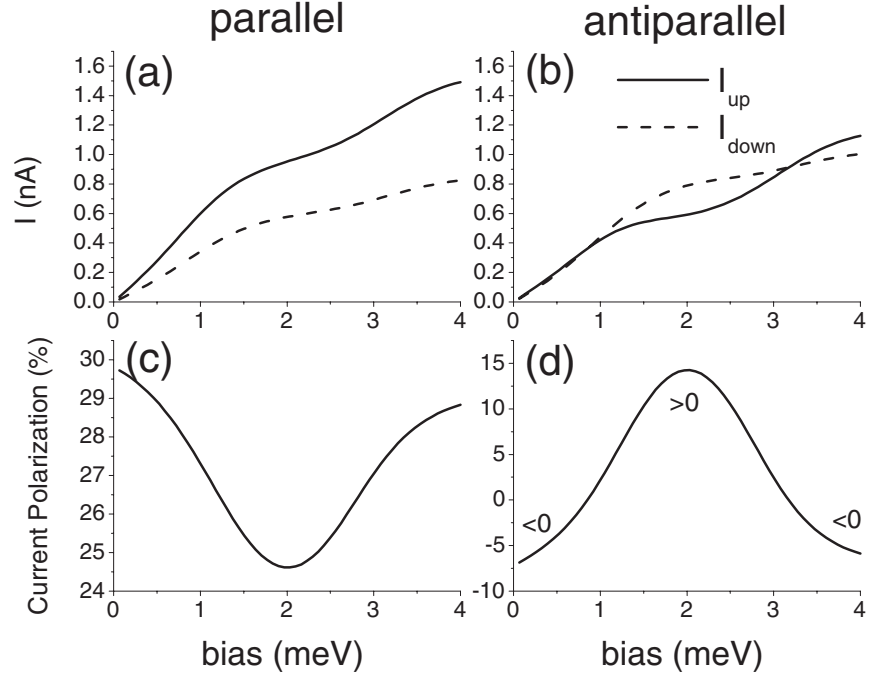

FIG. 3. [(a)-(b)] Spin-resolved currents $I_{\uparrow}$ and $I_{\downarrow}$ and [(c)-(d)] current polarization for both parallel and antiparallel alignments. In the $\mathrm{P}$ case we find $I_{\uparrow}>I_{\downarrow}$. In contrast, for AP alignment $I_{\uparrow}<I_{\downarrow}$ in the bias window 1-3 meV (singly occupied regime) and $I_{\uparrow}>I_{\downarrow}$ when the bias exceeds the charging energy above $3 \mathrm{meV}$ (doubly occupied regime). Panels (c)-(d) reveal a suppression and an enhancement of the current polarization in the singly occupied regime for the P and AP cases, respectively. Parameters: $p_{L}=0.23, p_{R}=0.35$, $k_{B} T=200 \mu \mathrm{eV}, \Gamma_{0}=10 \mu \mathrm{eV}, R=0, \epsilon_{0}=0.5 \mathrm{meV}$, and $U=1 \mathrm{meV}$.

$=0.23$. In contrast, $\wp^{R}$ assume negative values tending to $-p_{R}$ as $R$ increases. This shows that even though we have a constant total current along the system $\left(I_{L}^{\uparrow}+I_{L}^{\downarrow}=I_{R}^{\uparrow}+I_{R}^{\downarrow}\right)$, its polarization can change across the system when $R \neq 0$, i.e., when there is a transverse magnetic field applied on the dot, which coherently rotates the spin.

Figure 5 shows $I_{\eta}^{\sigma}$ against $R$ for $R=0, I_{L}^{\uparrow}=I_{R}^{\uparrow}$, and $I_{L}^{\downarrow}=I_{R}^{\downarrow}$ as expected. For $R \neq 0$ these equalities disappear, with $I_{L}^{\uparrow}$ and $I_{R}^{\downarrow}$ increasing and $I_{L}^{\downarrow}$ and $I_{R}^{\uparrow}$ decreasing with $R$. This leads to an

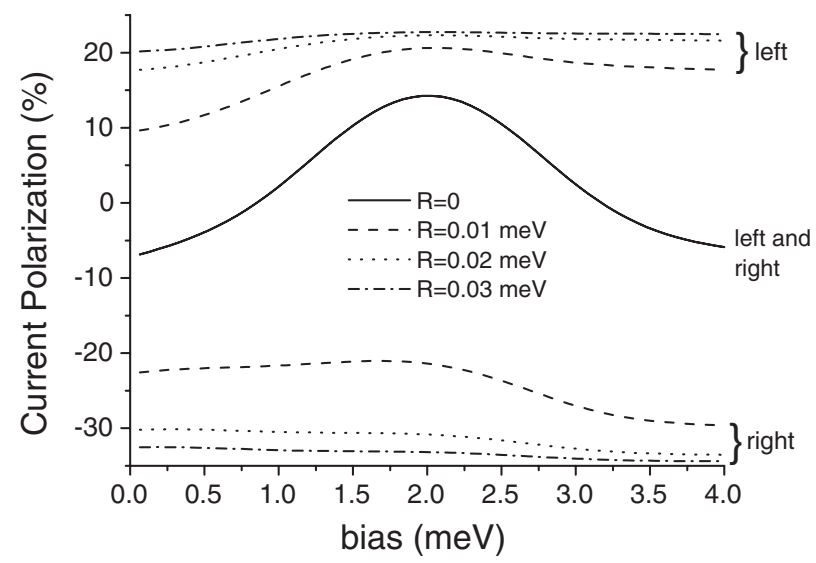

FIG. 4. Current polarization against the bias voltage for differing spin-flip rates in the AP alignment and in both left and right leads. For $R=0$ the polarization is the same on both sides. When $R \neq 0$, though, the current polarization in the left side enhances, tending to the left magnetization degree $p_{L}$. In contrast, the current polarization in the right side assumes negative values, tending to $-p_{R}$. Parameters: $p_{L}=0.23, p_{R}=0.35, k_{B} T=200 \mu \mathrm{eV}, \Gamma_{0}=10 \mu \mathrm{eV}$, $\epsilon_{0}=0.5 \mathrm{meV}$, and $U=1 \mathrm{meV}$. 


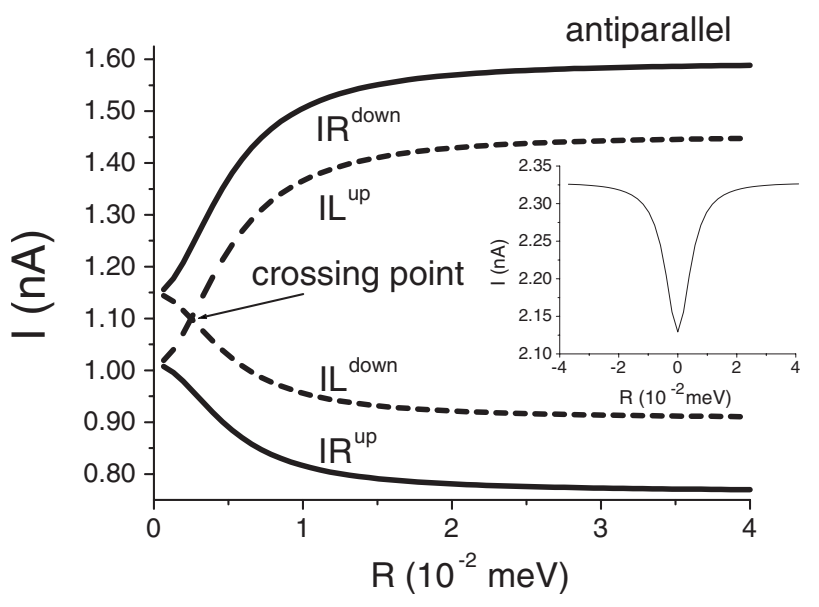

FIG. 5. Spin-resolved currents versus $R$. For $R=0$ we have $I_{L}^{\uparrow}$ $=I_{R}^{\uparrow}$ and $I_{L}^{\downarrow}=I_{R}^{\downarrow}$ with $I_{\eta}^{\uparrow}$ and $I_{\eta}^{\downarrow}$ being different from each other. For increasing $R, I_{L}^{\uparrow}$ and $I_{R}^{\downarrow}$ grow while $I_{L}^{\downarrow}$ and $I_{R}^{\uparrow}$ decreases. For a particular $R$ there is a crossing between $I_{L}^{\uparrow}$ and $I_{L}^{\downarrow}$, thus resulting simultaneously in a nonpolarized left current and a polarized right current. Parameters: $p_{L}=0.23, p_{R}=0.35, k_{B} T=200 \mu \mathrm{eV}, \Gamma_{0}=10 \mu \mathrm{eV}$, $\epsilon_{0}=0.5 \mathrm{meV}, U=1 \mathrm{meV}$, and bias $=4 \mathrm{meV}$.

enhancement of $\wp^{L}$ and $\left|\wp_{R}\right|$ as seen in Fig. 4. Interestingly, there is a crossing point between $I_{L}^{\uparrow}$ and $I_{L}^{\downarrow}$ around $R \approx \Gamma_{0} / 4$. So for this particular $R$ the total current becomes unpolarized in the emitter (left) lead and relatively high polarized in the collector (right) lead. This means that it is possible to change the current polarization from emitter to collector lead by precessing the electron spin in the quantum dot.

In the inset of Fig. 5 we show the total current against $R$. This curve resembles a typical Hanle resonance. ${ }^{36,37}$ Similar to Ref. 37, here we can say that in the AP configuration and positive bias (i.e., with left being the emitter) the dot tends to be more up populated due to the majority up population in the emitter and the majority down population in the collector lead. On average a transverse magnetic field tends to increase the spin-down component in the dot along the down magnetization of the collector lead. As a result, the electron can more easily tunnel into the right ferromagnet and the current increases.

\section{Shot noise}

Figure 6 shows the Fano factor, $\gamma=S_{L L} / 2 e I_{L}$, against $R$ in the AP configurations. The $\mathrm{P}$ alignment gives approximately insensitive Fano factor with respect to $R$. In the AP configuration, the Fano factor can be suppressed with $R$, reaching values below 0.5 . This suppression can be further intensified by increasing the lead-polarization parameters $p_{L}$ and $p_{R}$. In particular, for fully spin-polarized leads $(p=1)$ AP aligned, the Fano factor reaches values close to 0.3 when double occupancy is allowed (bias $=6 \mathrm{meV}$ ), and it attains 0.35 in the single-occupancy regime (bias $=2 \mathrm{meV}$ ). ${ }^{55}$ For fully polarized leads in the $\mathrm{P}$ configuration the Fano factor remains at 0.5 independently of $R$.

A simple physical picture for this additional suppression of $\gamma$ is as follows: Consider an up-spin sitting on the dot. A second up-spin trying to hop onto it is Pauli blocked until the

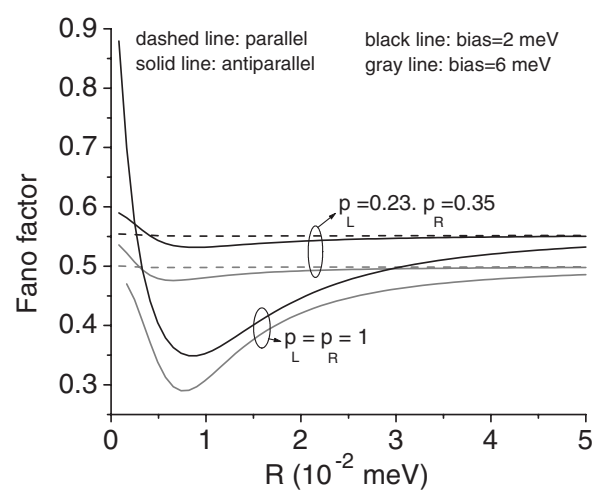

FIG. 6. Fano factor against $R$ for two fixed bias voltages corresponding to single (bias $=2 \mathrm{meV}$ ) and double occupancy (bias $=6 \mathrm{meV}$ ) in both $\mathrm{P}$ and AP alignments. In the P case the Fano factor is approximately constant. In contrast, in the AP alignment the Fano factor can be suppressed due to spin flip, reaching values below 0.5 . In particular, for fully polarized leads $\left(p_{L}=p_{R}=1\right)$ the Fano factor can be strongly suppressed, assuming values close to 0.3 when double occupancy is allowed. Parameters: $\epsilon_{0}=0.5 \mathrm{meV}$, $U=1 \mathrm{meV}, \Gamma_{0}=10 \mu \mathrm{eV}$, and $k_{B} T=200 \mu \mathrm{eV}$.

first electron tunnel to the collector lead or undergo a coherent spin flip. If the spin flip is fast enough (faster than the into/out tunneling processes), the first electron can return to its original state (via another spin flip) instead of tunneling out of the dot. This blocks additionally the second up-spin, consequently suppressing even further the noise.

We note that for any $R$ when we go from the single (bias $=2 \mathrm{meV}$ ) to the double (bias $=6 \mathrm{meV}$ ) occupation regimes a reduction in the Fano factor is observed in both $\mathrm{P}$ and AP alignments (cf. solid black to solid gray lines and dashed black to dashed gray lines). This general feature was already predicted in Ref. 56, where a diagrammatic formulation for the noise is derived. It is valid to mention that in the present study we have performed an $S$-matrix expansion (Appendix B) to the noise, which could in principle be mapped into a Feynman diagrammatic formulation. Comparing our results with previous findings in the literature, we observe a difference between them in the single-occupancy regime. ${ }^{52}$ Figure 7 shows a comparison for the shot noise obtained from Eq. (30) and from the analytical results in Ref. 54 (derived for $p_{L}=p_{R}=0$ and $R=0$ ). While the second plateau [II in Fig. 7] coincides in both numerical and analytical cases, the first plateaus (I in the plot) do not coincide. ${ }^{57}$ This disagreement is related to the Hartree-Fock factorization underlying our calculation.

We also note that without Coulomb interaction $(U=0)$ and for fully spin-polarized leads antiparallel aligned, the Fano factor is given by

$$
\gamma=1-\frac{1}{2} \frac{\beta^{2}\left(5+\beta^{2}\right)}{\left(1+\beta^{2}\right)^{2}},
$$

where $\beta=R / \Gamma_{0}$. Equation (34) is also found for a three tunneling barrier junction. ${ }^{58}$ Hence, for fully spin-polarized AP leads and nonvanishing spin flip, the FM-QD-FM setup resembles a three-barrier geometry. ${ }^{59,60}$ 


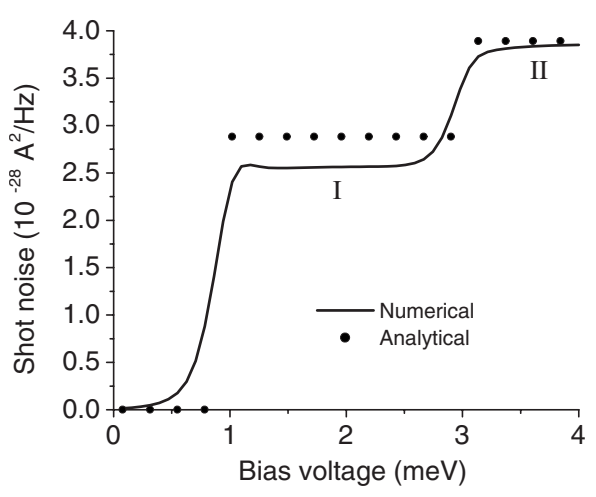

FIG. 7. Comparison of the shot noise for the present approach (numerical) and the one derived in Ref. 54 (analytical). While the second plateau (II) coincides in both approaches, the first plateau (i) differs. We attribute this contrast to the Hartree-Fock-type approximation used in our calculation. Parameters: $p_{L}=p_{R}=0, k_{B} T$ $=50 \mu \mathrm{eV}, \Gamma_{0}=10 \mu \mathrm{eV}, R=0, \epsilon_{0}=0.5 \mathrm{meV}$, and $U=1 \mathrm{meV}$.

\section{CONCLUSION}

Using the nonequilibrium Green's-function technique, we have studied the transport properties of a quantum dot coupled to two ferromagnetic leads. We consider both parallel (P) and antiparallel (AP) alignments of the lead polarizations. Coulomb interaction and coherent spin flip are included in our model. We find that for distinct ferromagnetic leads the interplay between Coulomb interaction and spin accumulation translates into an enhancement and a suppression of the current polarization $\wp$ in AP and $\mathrm{P}$ cases, respectively, depending on the bias. We also observe that the spin flip can change the current polarization when it flows from the emitter to the collector. It is even possible to have a polarized current in the collector while it is unpolarized in the emitter. We have derived an expression for the noise [Eq. (30)], which exactly accounts for spin flip but only approximately for Coulomb interaction. Finally, we found a suppression of the Fano factor to values well below 1/2 due to spin flip.

\section{ACKNOWLEDGMENTS}

The authors acknowledge support for this work from the Brazilian agencies CNPq, CAPES, FAPESP, and IBEM, and the FiDiPro program of the Finnish Academy. The authors thank J. König and J. Martinek for helpful discussions.

\section{APPENDIX A: DOT GREEN'S FUNCTIONS}

Here we present in some detail the calculation of the dot Green's functions, $\mathbf{G}^{r}, \mathbf{G}^{a}, \mathbf{G}^{>}$, and $\mathbf{G}^{<}$, used in the current and noise expressions. The starting point is to derive an equation of motion for the contour-ordered Green's function $G_{\sigma \sigma^{\prime}}\left(\tau, \tau^{\prime}\right)=-i\left\langle T_{c} d_{\sigma}(\tau) d_{\sigma^{\prime}}^{\dagger}\left(\tau^{\prime}\right)\right\rangle$, and then, via analytical continuation rules, to determine these Green's functions. After a straightforward calculation via equation of motion we find

$$
\begin{aligned}
\mathbf{G}\left(\tau, \tau^{\prime}\right)= & \mathbf{G}^{0}\left(\tau, \tau^{\prime}\right)+\iint d \tau_{1} d \tau_{2} \mathbf{G}^{0}\left(\tau, \tau_{1}\right) \Sigma\left(\tau_{1}, \tau_{2}\right) \mathbf{G}\left(\tau_{2}, \tau^{\prime}\right) \\
& +\int d \tau_{1} \mathbf{G}^{0}\left(\tau, \tau_{1}\right) U \mathbf{G}^{(2)}\left(\tau_{1}, \tau^{\prime}\right),
\end{aligned}
$$

where the components of $\mathbf{G}^{(2)}$ and $\boldsymbol{\Sigma}$ are

$$
G_{i j}^{(2)}\left(\tau, \tau^{\prime}\right)=-i\left\langle T_{c} n_{i}^{-}(\tau) d_{i}(\tau) d_{j}^{\dagger}\left(\tau^{\prime}\right)\right\rangle
$$

and

$$
\sum_{i j}\left(\tau, \tau^{\prime}\right)=\sum_{k \eta \sigma} \frac{1}{2}(-1)^{(i+j) \delta_{\sigma \downarrow}}\left|t_{k \sigma}\right|^{2} g_{k \sigma \eta}\left(\tau, \tau^{\prime}\right),
$$

respectively. $\mathbf{G}^{0}$ is the dot Green's function without both the coupling to leads and the Coulomb interaction.

Following the equation of motion expansion we find for $\mathbf{G}^{(2)}\left(\tau_{1}, \tau^{\prime}\right)$,

$$
\begin{aligned}
\mathbf{G}^{(2)}\left(\tau_{1}, \tau^{\prime}\right)= & \mathbf{G}^{0(2)}\left(\tau_{1}, \tau^{\prime}\right)+\iint d \tau_{2} d \tau_{3} \mathbf{G}^{0(2)}\left(\tau_{1}, \tau_{2}\right) \\
& \times \mathbf{\Sigma}\left(\tau_{2}, \tau_{3}\right) \mathbf{G}\left(\tau_{3}, \tau^{\prime}\right),
\end{aligned}
$$

where $\mathbf{G}^{0(2)}\left(\tau_{1}, \tau^{\prime}\right)$ satisfies the identity,

$$
\begin{aligned}
\left(i \frac{\partial}{\partial \tau}-\epsilon_{i}-U\right) G_{i j}^{0(2)}\left(\tau, \tau^{\prime}\right)= & \delta_{i j} \delta\left(t-t^{\prime}\right)\left\langle n_{i}^{-}\right\rangle \\
& -\delta_{i j}^{-} \delta\left(t-t^{\prime}\right)\left\langle d_{i}^{\dagger} d_{i}\right\rangle .
\end{aligned}
$$

In Eq. (A2) we have used the following approximations: ${ }^{61}$

$$
\begin{gathered}
\left\langle T d_{i}(t) d_{i}(t) c_{k \sigma \eta}^{\dagger}(t) d_{j}^{\dagger}\left(t^{\prime}\right)\right\rangle=0, \\
\left\langle T d_{i}^{\dagger}(t) d_{i}(t) c_{k \sigma \eta}(t) d_{j}^{\dagger}\left(t^{\prime}\right)\right\rangle=\left\langle d_{i}^{\dagger} d_{i}\right\rangle\left\langle T c_{k \sigma \eta}(t) d_{j}^{\dagger}\left(t^{\prime}\right)\right\rangle, \\
\left\langle T d_{i}^{\dagger}(t) d_{i}^{-}(t) c_{k \sigma \eta}(t) d_{j}^{\dagger}\left(t^{\prime}\right)\right\rangle=\left\langle d_{i}^{\dagger} d_{i}^{-}\right\rangle\left\langle T c_{k \sigma \eta}(t) d_{j}^{\dagger}\left(t^{\prime}\right)\right\rangle .
\end{gathered}
$$

Observe that Eq. (A2) closes the system of Eqs. (A1) and (A2). Substituting Eq. (A2) into Eq. (A1) we find a Dyson equation for $\mathbf{G}$,

$$
\begin{aligned}
\mathbf{G}\left(\tau, \tau^{\prime}\right)= & \widetilde{\mathbf{G}}^{0}\left(\tau, \tau^{\prime}\right)+\iint d \tau_{1} d \tau_{2} \widetilde{\mathbf{G}}^{0}\left(\tau, \tau_{1}\right) \\
& \times \mathbf{\Sigma}\left(\tau_{1}, \tau_{2}\right) \mathbf{G}\left(\tau_{2}, \tau^{\prime}\right),
\end{aligned}
$$

where

$$
\widetilde{\mathbf{G}}^{0}\left(\tau, \tau^{\prime}\right)=\mathbf{G}^{0}\left(\tau, \tau^{\prime}\right)+\int d \tau_{1} \mathbf{G}^{0}\left(\tau, \tau_{1}\right) U \mathbf{G}^{0(2)}\left(\tau_{1}, \tau^{\prime}\right)
$$

Applying analytical continuation rules into Eqs. (A4) and (A5), we find

$$
\begin{aligned}
\mathbf{G}^{r}\left(t, t^{\prime}\right)= & \widetilde{\mathbf{G}}^{0 r}\left(t, t^{\prime}\right)+\iint d t_{1} d t_{2} \widetilde{\mathbf{G}}^{0 r}\left(t, t_{1}\right) \\
& \times \boldsymbol{\Sigma}^{r}\left(t_{1}, t_{2}\right) \mathbf{G}^{r}\left(t_{2}, t^{\prime}\right),
\end{aligned}
$$




$$
\widetilde{\mathbf{G}}^{0 r}\left(t, t^{\prime}\right)=\mathbf{G}^{0 r}\left(t, t^{\prime}\right)+\int d t_{1} \mathbf{G}^{0 r}\left(t, t_{1}\right) U \mathbf{G}^{0(2)}\left(t_{1}, t^{\prime}\right),
$$

and also the Keldysh equation,

$$
\mathbf{G}^{<}\left(t, t^{\prime}\right)=\iint d t_{1} d t_{2} \mathbf{G}^{r}\left(t, t_{1}\right) \mathbf{\Sigma}^{<}\left(t_{1}, t_{2}\right) \mathbf{G}^{a}\left(t_{2}, t^{\prime}\right) .
$$

Via Fourier transform of Eqs. (A6) and (A7), we obtain

$$
\begin{gathered}
\mathbf{G}^{r}(\boldsymbol{\epsilon})=\left[\widetilde{\mathbf{G}}^{0 r^{-1}}(\boldsymbol{\epsilon})-\Sigma^{r}(\epsilon)\right]^{-1}, \\
\widetilde{\mathbf{G}}^{0 r}(\boldsymbol{\epsilon})=\mathbf{G}^{0 r}(\boldsymbol{\epsilon})+\mathbf{G}^{0 r}(\boldsymbol{\epsilon}) U \mathbf{G}^{0(2)}(\boldsymbol{\epsilon}),
\end{gathered}
$$

and of Eq. (A8) we find

$$
\mathbf{G}^{<}(\epsilon)=\mathbf{G}^{r}(\epsilon) \mathbf{\Sigma}^{<}(\epsilon) \mathbf{G}^{a}(\epsilon),
$$

where

$$
\mathbf{G}^{0 r}(\epsilon)=\left(\begin{array}{cc}
\frac{1}{\epsilon-\epsilon_{1}+i 0^{+}} & 0 \\
0 & \frac{1}{\epsilon-\epsilon_{2}+i 0^{+}}
\end{array}\right)
$$

and

$$
\mathbf{G}^{0(2) r}(\epsilon)=\left(\begin{array}{cc}
\frac{\left\langle n_{2}\right\rangle}{\epsilon-\epsilon_{1}-U+i 0^{+}} & -\frac{\left\langle d_{2}^{\dagger} d_{1}\right\rangle}{\epsilon-\epsilon_{1}-U+i 0^{+}} \\
-\frac{\left\langle d_{1}^{\dagger} d_{2}\right\rangle}{\epsilon-\epsilon_{2}-U+i 0^{+}} & \frac{\left\langle n_{1}\right\rangle}{\epsilon-\epsilon_{2}-U+i 0^{+}}
\end{array}\right) .
$$

The retarded self-energy is given by

$$
\Sigma^{r}=-\frac{i}{2}\left(\begin{array}{ll}
\Gamma_{\uparrow}+\Gamma_{\downarrow} & \Gamma_{\uparrow}-\Gamma_{\downarrow} \\
\Gamma_{\uparrow}-\Gamma_{\downarrow} & \Gamma_{\uparrow}+\Gamma_{\downarrow}
\end{array}\right),
$$

and the lesser self-energy is defined as

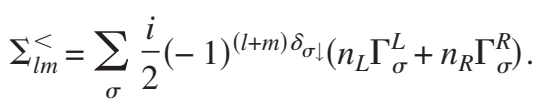

\section{APPENDIX B: $S$-MATRIX EXPANSION FOR THE NOISE}

According to Eq. (21) the noise is given in terms of the four-operator Green's functions $g_{i}^{(2)}\left(\tau, \tau^{\prime}\right)(i=1,2,3,4)$. To determine their equations of motion we develop an $S$-matrix expansion as we illustrate below for $g_{1}^{(2)}\left(\tau, \tau^{\prime}\right)$. The first step is to transform the operators from the Heisenberg to the interaction picture,

$$
g_{1}^{(2)}\left(\tau, \tau^{\prime}\right)=i^{2}\left\langle T_{c} S \widetilde{c}_{k \sigma \eta}^{\dagger}(\tau) \widetilde{d}_{i}(\tau) \tilde{c}_{k^{\prime} \sigma^{\prime} \eta^{\prime}}^{\dagger}\left(\tau^{\prime}\right) \tilde{d}_{j}\left(\tau^{\prime}\right)\right\rangle,
$$

where the tilde denotes the operators in the interaction picture, i.e.,

$$
d_{i}(t)=v^{\dagger}\left(t, t_{0}\right) \tilde{d}_{i}(t) v\left(t, t_{0}\right),
$$

with

$$
v\left(t, t_{0}\right)=T e^{-i \int_{t_{0}}^{t} d t^{\prime} \tilde{H}_{T}\left(t^{\prime}\right)},
$$

and a similar definition for the $c$ operator. The operator $T$ is the time-ordering operator. The $S$-matrix in $g_{1}^{(2)}\left(\tau, \tau^{\prime}\right)$ is defined as

$$
S=T_{c} e^{-i \int_{c} d \tau_{1} \tilde{H}_{T}\left(\tau_{1}\right)} .
$$

Expanding $S$ we find

$$
\begin{aligned}
g_{1}^{(2)}\left(\tau, \tau^{\prime}\right)= & i^{2} \sum_{n=0}^{\infty} \frac{(-i)^{n}}{n !} \\
& \times\left\langle T_{c} \widetilde{c}_{k \sigma \eta}^{\dagger}(\tau) \widetilde{d}_{i}(\tau) \tilde{c}_{k^{\prime} \sigma^{\prime} \eta^{\prime}}^{\dagger}\left(\tau^{\prime}\right) \tilde{d}_{j}\left(\tau^{\prime}\right)\right. \\
& \times\left[\int d \tau _ { 1 } \sum _ { k _ { 1 } \sigma _ { 1 } \eta _ { 1 } i _ { 1 } } \frac { ( - 1 ) ^ { i _ { 1 } \delta _ { \sigma _ { 1 } \downarrow } } } { \sqrt { 2 } } \left(t_{k_{1} \sigma_{1}} \widetilde{c}_{k_{1} \sigma_{1} \eta_{1}}^{\dagger}\left(\tau_{1}\right)\right.\right. \\
& \left.\left.\left.\times \widetilde{d}_{i_{1}}\left(\tau_{1}\right)\right)+t_{k_{1} \sigma_{1}}^{*} \widetilde{d}_{i_{1}}^{\dagger}\left(\tau_{1}\right) \widetilde{c}_{k_{1} \sigma_{1} \eta_{1}}\left(\tau_{1}\right)\right]^{n}\right\rangle,
\end{aligned}
$$

where the lowest-order nonzero term in the expansion is that of $n=2$. Since we assume noninteracting leads, we can factorize the angle bracket in Eq. (B4) into a product of the lead and dot parts. We then apply Wick's theorem to the lead part. This results in

$$
\begin{aligned}
g_{1}^{(2)}\left(\tau, \tau^{\prime}\right)= & t_{k \sigma}^{*} t_{k^{\prime} \sigma^{\prime}}^{*} \iint d \tau_{1} d \tau_{2} \\
& \times \sum_{k_{1} \sigma_{1} \eta_{1}} \sum_{k_{2} \sigma_{2} \eta_{2}} \sum_{i_{1} i_{2}=1,2} \frac{1}{2}(-1)^{i_{1} \delta_{\sigma_{1} \downarrow}}(-1)^{i_{2} \delta_{\sigma_{2} \downarrow} \downarrow}(-i) \\
& \times\left\langle T_{c} \widetilde{c}_{k_{1} \sigma_{1} \eta_{1}}\left(\tau_{1}\right) \widetilde{c}_{k \sigma \eta}^{\dagger}(\tau)\right\rangle(-i) \\
& \times\left\langle T_{c} \widetilde{c}_{k_{2} \sigma_{2} \eta_{2}}\left(\tau_{2}\right) \widetilde{c}_{k^{\prime} \sigma^{\prime} \eta^{\prime}}^{\dagger}\left(t^{\prime}\right)\right\rangle \\
& \times\left\langle T_{c} S \widetilde{d}_{i}(\tau) \tilde{d}_{j}\left(\tau^{\prime}\right) \tilde{d}_{i_{1}}^{\dagger}\left(\tau_{1}\right) \tilde{d}_{i_{2}}^{\dagger}\left(\tau_{2}\right)\right\rangle .
\end{aligned}
$$

In Eq. (B5) we have contracted $\widetilde{c}_{k_{1} \sigma_{1} \eta_{1}}$ with $\widetilde{c}_{k \sigma \eta}^{\dagger}$, and $\widetilde{c}_{k_{2} \sigma_{2} \eta_{2}}$ with $\widetilde{c}_{k^{\prime} \sigma^{\prime} \eta^{\prime}}^{\dagger}$. This is one choice among $n(n-1)$ possible contractions. Since all of them yield the same result, we simply multiply the chosen pairing by $n(n-1)$. This factor cancels part of the factorial $n$ ! in Eq. (B4), thus resulting in the $S$ matrix in the last angle bracket of Eq. (B5).

The first and second averages in Eq. (B5) give $\delta_{k \sigma \eta, k_{1} \sigma_{1} \eta_{1}}$ and $\delta_{k^{\prime} \sigma^{\prime} \eta^{\prime}, k_{2}, \sigma_{2}, \eta_{2}}$, respectively, so the sums over $\left(k_{1}, \sigma_{1}, \eta_{1}\right)$ and $\left(k_{2}, \sigma_{2}, \eta_{2}\right)$ disappear. Defining $g_{k \sigma \eta}\left(\tau_{1}, \tau\right)=$ $-i\left\langle T_{c} \widetilde{c}_{k \sigma \eta}\left(\tau_{1}\right) c_{k \sigma \eta}^{\dagger}(\tau)\right\rangle$ and $g_{k^{\prime} \sigma^{\prime} \eta^{\prime}}\left(\tau^{\prime}, \tau_{2}\right)=$ $-i\left\langle T_{c} \widetilde{c}_{k^{\prime} \sigma^{\prime} \eta^{\prime}}\left(\tau^{\prime}\right) \widetilde{c}_{k^{\prime} \sigma^{\prime} \eta^{\prime}}^{\prime}\left(\tau_{2}\right)\right\rangle$, we can rewrite Eq. (B5) as

$$
\begin{aligned}
g_{1}^{(2)}\left(\tau, \tau^{\prime}\right)= & t_{k \sigma^{\prime}}^{*} t_{k^{\prime} \sigma^{\prime}}^{*} \iint d \tau_{1} d \tau_{2} \\
& \times \sum_{i_{1}, i_{2}=1,2} \frac{1}{2}(-1)^{i_{1} \delta_{\sigma_{1} \downarrow}}(-1)^{i_{2} \delta_{\sigma_{2} \downarrow}} \\
& \times g_{k \sigma \eta}\left(\tau_{1}, \tau\right) g_{k^{\prime} \sigma^{\prime} \eta^{\prime}}\left(\tau_{2}, \tau^{\prime}\right) \\
& \times\left\langle T_{c} d_{i}(\tau) d_{j}\left(\tau^{\prime}\right) d_{i_{1}}^{\dagger}\left(\tau_{1}\right) d_{i_{2}}^{\dagger}\left(\tau_{2}\right)\right\rangle .
\end{aligned}
$$


For the $U=0$ case the calculation is straightforward. By applying Wick's theorem in the four-operator Green's function, we find

$$
\begin{aligned}
g_{1}^{(2)}\left(\tau, \tau^{\prime}\right)= & t_{k \sigma}^{*} t_{k^{\prime} \sigma^{\prime}}^{*} \iint d \tau_{1} d \tau_{2} \\
& \times \sum_{i_{1} i_{2}} \frac{1}{2}(-1)^{i_{1} \delta_{\sigma \downarrow}(-1)^{i_{2} \delta_{\sigma^{\prime} \downarrow}}} \\
& \times g_{k \sigma \eta}\left(\tau_{1}, \tau\right) g_{k^{\prime} \sigma^{\prime} \eta^{\prime}}\left(\tau_{2}, \tau^{\prime}\right) \\
& \times\left\{G_{i i_{1}}\left(\tau, \tau_{1}\right) G_{j i_{2}}\left(\tau^{\prime}, \tau_{2}\right)-G_{i i_{2}}\left(\tau, \tau_{2}\right) G_{j i_{1}}\left(\tau^{\prime}, \tau_{1}\right)\right\},
\end{aligned}
$$

where $G_{i i_{1}}\left(\tau, \tau_{1}\right)=-i\left\langle T_{c} d_{i}(\tau) d_{i_{1}}^{\dagger}\left(\tau_{1}\right)\right\rangle$, plus analogous definitions for the other Green's functions. A similar calculation yields Eq. (23) for $g_{2}^{(2)}\left(\tau, \tau^{\prime}\right)$. In the presence of the Coulomb interaction $(U \neq 0)$ Eq. (B6) is no longer exact and the full diagrammatic expansion should be considered in order to find an accurate noise expression. However, this is a formidable task since it involves not only the usual many body expansion but also the analytical continuation of two and more particles Green's functions. So as a first approximation, we use Eq. (B6) even in the presence of the Coulomb interaction.

\section{APPENDIX C: "LINKED-CLUSTER THEOREM" FOR THE NOISE EXPANSION}

Equations (22)-(25) are composed of what we call connected and disconnected terms. Here we show that the disconnected parts cancel identically the term $2 I_{\eta}^{2}$ in Eq. (21). Writing explicitly the disconnected term of Eq. (22), we have

$$
\begin{aligned}
g_{1 \mathrm{disc}}^{(2)}\left(\tau, \tau^{\prime}\right)= & t_{k \sigma}^{*} t_{k^{\prime} \sigma^{\prime}}^{*} \sum_{i_{1} i_{2}} \frac{1}{2}(-1)^{i_{1} \delta_{\sigma \downarrow}(-1)^{i_{2} \delta_{\sigma^{\prime} \downarrow}}} \\
& \times \int d \tau_{1} G_{i i_{1}}\left(\tau, \tau_{1}\right) g_{k \sigma \eta}\left(\tau_{1}, \tau^{+}\right) \\
& \times \int d \tau_{2} G_{j i_{2}}\left(\tau^{\prime}, \tau_{2}\right) g_{k^{\prime} \sigma^{\prime} \eta^{\prime}}\left(\tau_{2}, \tau^{\prime+}\right),
\end{aligned}
$$

where the + sign on one of the $\tau$ and $\tau^{\prime}$ is just a reminder that the sequence of operators $c_{k \sigma \eta}^{\dagger}(\tau) d_{i}(\tau)$ and $c_{k^{\prime} \sigma^{\prime} \eta^{\prime}}^{\dagger}\left(\tau^{\prime}\right) d_{j}\left(\tau^{\prime}\right)$ in the main definition of $g_{1}^{(2)}\left(\tau, \tau^{\prime}\right)$ (beginning of Sec. III D) should be preserved during the following calculation. Applying the analytic continuation rules, we obtain

$$
g_{\text {disc }}^{(2)>>}\left(t, t^{\prime}\right)=t_{k \sigma^{\prime}}^{*} t_{k^{\prime} \sigma^{\prime}}^{*} F_{i \sigma, k \eta \sigma}(t, t) F_{j \sigma^{\prime}, k^{\prime} \eta^{\prime} \sigma^{\prime}}\left(t^{\prime}, t^{\prime}\right),
$$

with

$$
\begin{aligned}
F_{i \sigma, k \eta \sigma}(t, t)= & \sum_{i_{1}} \frac{1}{\sqrt{2}}(-1)^{i_{1} \delta_{\sigma \downarrow}} \int d t_{1}\left[G_{i i_{1}}^{r}\left(t, t_{1}\right) g_{k \sigma \eta}^{<}\left(t_{1}, t\right)\right. \\
& \left.+G_{i i_{1}}^{<}\left(t, t_{1}\right) g_{k \sigma \eta}^{a}\left(t_{1}, t\right)\right],
\end{aligned}
$$

and a similar definition for $F_{j \sigma^{\prime}, k^{\prime} \eta^{\prime} \sigma^{\prime}}\left(t^{\prime}, t^{\prime}\right)$. Similarly, from Eq. (23), we have

$$
\begin{aligned}
g_{2 \mathrm{disc}}^{(2)}\left(\tau, \tau^{\prime}\right)= & t_{k \sigma}^{*} t_{k^{\prime} \sigma^{\prime}} \sum_{i_{1}, i_{2}=1,2} \frac{1}{2}(-1)^{i_{1} \delta_{\sigma \downarrow}(-1)^{i_{2} \delta_{\sigma^{\prime} \downarrow}}} \\
& \times \int d \tau_{1} G_{i i_{1}}\left(\tau, \tau_{1}\right) g_{k \sigma \eta}\left(\tau_{1}, \tau^{+}\right) \\
& \times \int d \tau_{2} g_{k^{\prime} \sigma^{\prime} \eta^{\prime}}\left(\tau^{\prime}, \tau_{2}\right) G_{i_{2} j}\left(\tau_{2}, \tau^{\prime+}\right) .
\end{aligned}
$$

which, after analytic continuation, can be expressed as

$$
g_{2 \mathrm{disc}}^{(2)>}\left(t, t^{\prime}\right)=-t_{k \sigma}^{*} t_{k^{\prime} \sigma^{\prime}} F_{i \sigma, k \eta \sigma}(t, t) F_{j \sigma^{\prime}, k^{\prime} \eta^{\prime} \sigma^{\prime}}^{*}\left(t^{\prime}, t^{\prime}\right) .
$$

Using the identities Eqs. (24) and (25) we obtain

$$
g_{3 \mathrm{disc}}^{(2)>}\left(t, t^{\prime}\right)=-t_{k \sigma} t_{k^{\prime} \sigma^{\prime}}^{*} F_{i \sigma, k \eta \sigma}^{*}(t, t) F_{j \sigma^{\prime}, k^{\prime} \eta^{\prime} \sigma^{\prime}}\left(t^{\prime}, t^{\prime}\right)
$$

and

$$
g_{4 \mathrm{disc}}^{(2)>}\left(t, t^{\prime}\right)=t_{k \sigma} t_{k^{\prime} \sigma^{\prime}} F_{i \sigma, k \eta \sigma}^{*}(t, t) F_{j \sigma^{\prime}, k^{\prime} \eta^{\prime} \sigma^{\prime}}^{*}\left(t^{\prime}, t^{\prime}\right) .
$$

From Eqs. (19) and (21) we note that

$$
\begin{aligned}
\left\langle\left\{\hat{I}_{\eta}(t), \hat{I}_{\eta^{\prime}}\left(t^{\prime}\right)\right\}\right\rangle_{\mathrm{disc}}= & e^{2} \sum_{k k^{\prime} \sigma \sigma^{\prime} i j} \frac{1}{2}(-1)^{i \delta_{\sigma \downarrow}(-1)^{j} \delta_{\sigma^{\prime} \downarrow}} \\
& \times\left\{t_{k \sigma} t_{k^{\prime} \sigma^{\prime}} g_{1 \mathrm{disc}}^{(2)>>}\left(t, t^{\prime}\right)-t_{k \sigma} t_{k^{\prime} \sigma^{\prime}}^{*} g_{2 \mathrm{disc}}^{(2)>}\left(t, t^{\prime}\right)\right. \\
& \left.-t_{k \sigma}^{*} t_{k^{\prime} \sigma^{\prime}} g_{\text {3disc }}^{(2)>}\left(t, t^{\prime}\right)+t_{k \sigma}^{*} t_{k^{\prime} \sigma^{\prime}}^{*} g_{4 \mathrm{disc}}^{(2)>}\left(t, t^{\prime}\right)\right\} \\
& + \text { H.c. }
\end{aligned}
$$

Using Eqs. (C1), (C4), (C6), and (C7) into Eq. (C8) we find

$$
\begin{aligned}
& \left\langle\left\{\hat{I}_{\eta}(t), \hat{I}_{\eta^{\prime}}\left(t^{\prime}\right)\right\}\right\rangle_{\mathrm{disc}}=2 e^{2} \sum_{k k^{\prime} \sigma \sigma^{\prime} i j}\left|t_{k \sigma}\right|^{2}\left|t_{k^{\prime} \sigma^{\prime}}\right|^{2} \frac{1}{2}(-1)^{i \delta_{\sigma \downarrow}}(-1)^{j \delta_{\sigma^{\prime} \downarrow}} \\
& \times\left[F_{i \sigma, k \eta \sigma}(t, t)+F_{i \sigma, k \eta \sigma}^{*}(t, t)\right] \\
& \times\left[F_{j \sigma^{\prime}, k^{\prime} \eta^{\prime} \sigma^{\prime}}\left(t^{\prime}, t^{\prime}\right)+F_{j \sigma^{\prime}, k^{\prime} \eta^{\prime} \sigma^{\prime}}^{*}\left(t^{\prime}, t^{\prime}\right)\right] .
\end{aligned}
$$

On the other hand we can write the current as

$$
\begin{gathered}
\left\langle\hat{I}_{\eta}(t)\right\rangle=2 e \operatorname{Re} \sum_{k \sigma i}\left|t_{k \sigma}\right|^{2} \frac{(-1)^{i \delta_{\sigma \downarrow}}}{\sqrt{2}} F_{i \sigma, k \eta \sigma}(t, t) \\
=\sum_{k \sigma i}\left|t_{k \sigma}\right|^{2} \frac{(-1)^{i \delta_{\sigma \downarrow}}}{\sqrt{2}}\left[F_{i \sigma, k \eta \sigma}(t, t)+F_{i \sigma, k \eta \sigma}^{*}(t, t)\right] .
\end{gathered}
$$

Squaring Eq. (C10) and multiplying it by two, we find

$$
\begin{aligned}
2\left\langle\hat{I}_{\eta}(t)\right\rangle\left\langle\hat{I}_{\eta^{\prime}}\left(t^{\prime}\right)\right\rangle= & 2 e^{2} \sum_{k k^{\prime} \sigma \sigma^{\prime} i j}\left|t_{k \sigma}\right|^{2}\left|t_{k^{\prime} \sigma^{\prime}}\right|^{2} \\
& \times \frac{1}{2}(-1)^{i \delta_{\sigma \downarrow}}(-1)^{j \delta_{\sigma^{\prime} \downarrow}} \\
& \times\left[F_{i \sigma, k \eta \sigma}(t, t)+F_{i \sigma, k \eta \sigma^{*}}^{*}(t, t)\right] \\
& \times\left[F_{j \sigma^{\prime}, k^{\prime} \eta^{\prime} \sigma^{\prime}}\left(t^{\prime}, t^{\prime}\right)+F_{j \sigma^{\prime}, k^{\prime} \eta^{\prime} \sigma^{\prime}}^{*}\left(t^{\prime}, t^{\prime}\right)\right] .
\end{aligned}
$$

Hence, Eq. (C11) cancels identically with Eq. (C9), i.e., 


$$
\left\langle\left\{\hat{I}_{\eta}(t), \hat{I}_{\eta^{\prime}}\left(t^{\prime}\right)\right\}\right\rangle_{\mathrm{disc}}-2\left\langle\hat{I}_{\eta}(t)\right\rangle\left\langle\hat{I}_{\eta^{\prime}}\left(t^{\prime}\right)\right\rangle=0 .
$$

\section{APPENDIX D: RECOVERING THE STANDARD FORMULA FOR THE NOISE}

To prove Eq. (31) we note that the Green's functions appearing in Eq. (30) can be written as follows:

$$
\begin{gathered}
\Gamma^{L} \mathbf{G}^{<}=i \Gamma^{L} \mathbf{G}^{r}\left(n_{L} \Gamma^{L}+n_{R} \Gamma^{R}\right) \mathbf{G}^{a}, \\
\Gamma^{L} \mathbf{G}^{>}=-i \Gamma^{L} \mathbf{G}^{r}\left[\left(1-n_{L}\right) \Gamma^{L}+\left(1-n_{R}\right) \Gamma^{R}\right] \mathbf{G}^{a}, \\
\Gamma^{L}\left(\mathbf{G}^{r}-\mathbf{G}^{a}\right)=-i \Gamma^{L} \mathbf{G}^{r}\left(\Gamma^{L}+\Gamma^{R}\right) \mathbf{G}^{a}, \\
\operatorname{Tr}\left[\Gamma^{L} \mathbf{G}^{a} \Gamma^{L} \mathbf{G}^{a}+\Gamma^{L} \mathbf{G}^{r} \Gamma^{L} \mathbf{G}^{r}\right] \\
=\operatorname{Tr}\left[2 \Gamma^{L} \mathbf{G}^{r} \Gamma^{L} \mathbf{G}^{a}+\Gamma^{L}\left(\mathbf{G}^{r}-\mathbf{G}^{a}\right) \Gamma^{L}\left(\mathbf{G}^{r}-\mathbf{G}^{a}\right)\right] .
\end{gathered}
$$

Now, defining the generalized transmission coefficients,

$$
\begin{aligned}
& \mathbf{T}_{L L}=\Gamma^{L} \mathbf{G}^{r} \Gamma^{L} \mathbf{G}^{a}, \\
& \mathbf{T}_{L R}=\Gamma^{L} \mathbf{G}^{r} \Gamma^{R} \mathbf{G}^{a},
\end{aligned}
$$

we can write the above set of equations [Eqs. (D1)-(D4)] in terms of $\mathbf{T}_{L L}$ and $\mathbf{T}_{L R}$,

$$
\Gamma^{L} \mathbf{G}^{<}=i n_{L} \mathbf{T}_{L L}+i n_{R} \mathbf{T}_{L R},
$$

$$
\begin{gathered}
\Gamma^{L} \mathbf{G}^{>}=-i\left(1-n_{L}\right) \mathbf{T}_{L L}-i\left(1-n_{R}\right) \mathbf{T}_{L R}, \\
\Gamma^{L}\left(\mathbf{G}^{r}-\mathbf{G}^{r}\right)=-i \mathbf{T}_{L L}-i \mathbf{T}_{L R}, \\
\operatorname{Tr}\left[\Gamma^{L} \mathbf{G}^{a} \Gamma^{L} \mathbf{G}^{a}+\Gamma^{L} \mathbf{G}^{r} \Gamma^{L} \mathbf{G}^{r}\right] \\
=\operatorname{Tr}\left[2 \mathbf{T}_{L L}-\left(\mathbf{T}_{L L}+\mathbf{T}_{L R}\right)\left(\mathbf{T}_{L L}+\mathbf{T}_{L R}\right)\right] .
\end{gathered}
$$

Using Eqs. (D7)-(D10) in Eq. (30) we obtain

$$
\begin{aligned}
S_{L L}(\omega=0)= & \frac{e^{2}}{\pi} \int d \epsilon \operatorname{Tr}\left\{n_{L}\left(1-n_{L}\right) \mathbf{T}_{L L}+n_{L}\left(1-n_{R}\right) \mathbf{T}_{L R}\right. \\
& +\left(1-n_{L}\right) n_{L} \mathbf{T}_{L L}+\left(1-n_{L}\right) n_{R} \mathbf{T}_{L R}+\left(n_{L} \mathbf{T}_{L L}\right. \\
& \left.+n_{R} \mathbf{T}_{L R}\right)\left[\left(1-n_{L}\right) \mathbf{T}_{L L}+\left(1-n_{R}\right) \mathbf{T}_{L R}\right] \\
& -n_{L}\left(\mathbf{T}_{L L}+\mathbf{T}_{L R}\right)\left[\left(1-n_{L}\right) \mathbf{T}_{L L}+\left(1-n_{R}\right) \mathbf{T}_{L R}\right] \\
& -\left(1-n_{L}\right)\left(\mathbf{T}_{L L}+\mathbf{T}_{L R}\right)\left[n_{L} \mathbf{T}_{L L}+n_{R} \mathbf{T}_{L R}\right]-n_{L}(1 \\
& \left.\left.-n_{L}\right)\left[2 \mathbf{T}_{L L}-\left(\mathbf{T}_{L L}+\mathbf{T}_{L R}\right)\left(\mathbf{T}_{L L}+\mathbf{T}_{L R}\right)\right]\right\} .
\end{aligned}
$$

The terms with $\mathbf{T}_{L L}$ cancel out identically and the above expression can be written as

$$
\begin{aligned}
S_{L L}(\omega=0)= & \frac{e^{2}}{\pi} \int d \boldsymbol{\epsilon} \times \operatorname{Tr}\left\{\left[n_{L}\left(1-n_{L}\right)+n_{R}\left(1-n_{R}\right)\right] \mathbf{T}_{L R}(\boldsymbol{\epsilon})\right. \\
& \left.+\left(n_{L}-n_{R}\right)^{2} \mathbf{T}_{L R}(\boldsymbol{\epsilon})\left[1-\mathbf{T}_{L R}(\boldsymbol{\epsilon})\right]\right\} . \quad \text { (D11) }
\end{aligned}
$$

Denoting $\mathbf{T}_{L R}$ simply as $\mathbf{T}$ we arrive at Eq. (31).
${ }^{1}$ S. Datta and B. Das, Appl. Phys. Lett. 56, 665 (1990).

${ }^{2}$ S. Cortez, O. Krebs, S. Laurent, M. Senes, X. Marie, P. Voisin, R. Ferreira, G. Bastard, J.-M. Gérard, and T. Amand, Phys. Rev. Lett. 89, 207401 (2002).

${ }^{3}$ M. Kroutvar, Y. Ducommun, D. Heiss, M. Bichler, D. Schuh, G. Abstreiter, and J. J. Finley, Nature (London) 432, 81 (2004).

${ }^{4}$ D. D. Awschalom, D. Loss, and N. Samarth, Semiconductor Spintronics and Quantum Computation (Springer-Verlag, Berlin, 2002).

${ }^{5}$ J. M. Elzerman, R. Hanson, L. H. W. van Beveren, B. Witkamp, L. M. K. Vandersypen, and L. P. Kouwenhoven, Nature (London) 430, 431 (2004).

${ }^{6}$ A. C. Johnson, J. R. Petta, J. M. Taylor, A. Yacoby, M. D. Lukin, C. M. Marcus, M. P. Hanson, and A. C. Gossard, Nature (London) 435, 925 (2005).

${ }^{7}$ F. H. L. Koppens, C. Buizert, K. J. Tielrooij, I. T. Vink, K. C. Nowack, T. Meunier, L. P. Kouwenhoven, and L. M. K. Vandersypen, Nature (London) 442, 766 (2006).

${ }^{8}$ M. A. Nielsen and I. L. Chuang, Quantum Computation and Quantum Information (Cambridge University Press, Cambridge, England, 2000).

${ }^{9}$ M. Jullière, Phys. Lett. 54A, 225 (1975).

${ }^{10}$ J. S. Moodera, L. R. Kinder, T. M. Wong, and R. Meservey, Phys. Rev. Lett. 74, 3273 (1995).

${ }^{11}$ M. N. Baibich, J. M. Broto, A. Fert, F. Nguyen Van Dau, F. Petroff, P. Eitenne, G. Creuzet, A. Friederich, and J. Chazelas,
Phys. Rev. Lett. 61, 2472 (1988).

${ }^{12}$ K. Hamaya, S. Masubuchi, M. Kawamura, T. Machida, M. Jung, K. Shibata, K. Hirakawa, T. Taniyama, S. Ishida, and Y. Arakawa, Appl. Phys. Lett. 90, 053108 (2007).

${ }^{13}$ K. Hamaya, M. Kitabatake, K. Shibata, M. Jung, M. Kawamura, K. Hirakawa, T. Machida, T. Taniyama, S. Ishida, and Y. Arakawa, Appl. Phys. Lett. 91, 022107 (2007).

${ }^{14}$ A. N. Pasupathy, R. C. Bialczak, J. Martinek, J. E. Grose, L. A. K. Donev, P. L. McEuen, and D. C. Ralph, Science 306, 86 (2004).

${ }^{15}$ S. Sahoo, T. Kontos, J. Furer, C. Hoffmann, M. Gräber, A. Cottet, and C. Schönenberger, Nat. Phys. 1, 99 (2005).

${ }^{16}$ C. A. Merchant and N. Marković, Phys. Rev. Lett. 100, 156601 (2008).

${ }^{17}$ I. Weymann and J. Barnaś, Phys. Rev. B 73, 033409 (2006).

${ }^{18}$ I. Weymann, J. König, J. Martinek, J. Barnaś, and G. Schön, Phys. Rev. B 72, 115334 (2005).

${ }^{19}$ F. M. Souza, J. C. Egues, and A. P. Jauho, Phys. Rev. B 75, 165303 (2007).

${ }^{20}$ This effect has been experimentally observed in Ref. 16 .

${ }^{21}$ F. Elste and C. Timm, Phys. Rev. B 73, 235305 (2006).

${ }^{22}$ A. Cottet, W. Belzig, and C. Bruder, Phys. Rev. Lett. 92, 206801 (2004).

${ }^{23}$ A. Cottet and W. Belzig, Europhys. Lett. 66, 405 (2004).

${ }^{24}$ M. Pioro-Ladrière, M. Ciorga, J. Lapointe, P. Zawadzki, M. Korkusiński, P. Hawrylak, and A. S. Sachrajda, Phys. Rev. Lett. 
91, 026803 (2003).

${ }^{25}$ F. M. Souza, Phys. Rev. B 76, 205315 (2007).

${ }^{26}$ E. Perfetto, G. Stefanucci, and M. Cini, Phys. Rev. B 78, 155301 (2008); G. Stefanucci, E. Perfetto, and M. Cini, ibid. 78, 075425 (2008).

${ }^{27}$ B. R. Bulka, J. Martinek, G. Michalek, and J. Barnaś, Phys. Rev. B 60, 12246 (1999).

${ }^{28}$ Y. Tserkovnyak and A. Brataas, Phys. Rev. B 64, 214402 (2001).

${ }^{29}$ E. G. Mishchenko, Phys. Rev. B 68, 100409(R) (2003).

${ }^{30}$ M. Zareyan and W. Belzig, Phys. Rev. B 71, 184403 (2005).

${ }^{31}$ R. Lopez and D. Sanchez, Phys. Rev. Lett. 90, 116602 (2003).

${ }^{32}$ F. M. Souza, J. C. Egues, and A. P. Jauho, arXiv:cond-mat/ 0209263, in Proceedings of the 26th International Conference on the Physics of Semiconductors (ICPS 26), Edinburgh, 2002, edited by J. H. Davies and A. R. Long.

${ }^{33}$ D. Urban, M. Braun, and J. König, Phys. Rev. B 76, 125306 (2007).

${ }^{34}$ A similar term has been used by a variety of authors describing spin-flip process in quantum dots; see, for instance, P. Zhang, Q.-K. Xue, and X. C. Xie, Phys. Rev. Lett. 91, 196602 (2003), and Refs. 26, 31, and 49.

${ }^{35}$ H.-A. Engel and D. Loss, Phys. Rev. Lett. 86, 4648 (2001).

${ }^{36}$ R. J. Epstein, D. T. Fuchs, W. V. Schoenfeld, P. M. Petroff, and D. D. Awschalom, Appl. Phys. Lett. 78, 733 (2001); L. Y. Zhang, C. Y. Wang, Y. G. Wei, X. Y. Liu, and D. Davidović, Phys. Rev. B 72, 155445 (2005).

${ }^{37}$ M. Braun, J. König, and J. Martinek, Europhys. Lett. 72, 294 (2005).

${ }^{38}$ Y. Meir and N. S. Wingreen, Phys. Rev. Lett. 68, 2512 (1992).

${ }^{39}$ A. P. Jauho, N. S. Wingreen, and Y. Meir, Phys. Rev. B 50, 5528 (1994).

${ }^{40}$ L. V. Keldysh, Sov. Phys. JETP 20, 1018 (1965).

${ }^{41}$ For a text-book treatment, see, e.g., H. Haug and A. P. Jauho, Quantum Kinetics in Transport and Optics of Semiconductors, Solid-State Sciences Vol. 123 (Springer, New York, 1996).

${ }^{42}$ M. Braun, J. König, and J. Martinek, Superlattices Microstruct. 37, 333 (2005)

${ }^{43}$ For a review see Ya. M. Blanter and M. Büttiker, Phys. Rep. 336, 1 (2000).

${ }^{44} \mathrm{~A}$ formally equivalent version of Eq. (30) has been published in the context of quantum dots with a phonon mode and Kondo physics; see J. X. Zhu and A. V. Balatsky, Phys. Rev. B 67, 165326 (2003); B. Dong and X. L. Lei, J. Phys.: Condens. Matter 14, 4963 (2002), respectively.

${ }^{45}$ G. B. Lesovik, Pis'ma Zh. Eksp. Teor. Fiz. 49, 513 (1989) [JETP
Lett. 49, 592 (1989)]; V. A. Khlus, Zh. Eksp. Teor. Fiz. 93, 2179 (1987) [Sov. Phys. JETP 66, 1243 (1987)].

${ }^{46}$ M. Büttiker, Phys. Rev. Lett. 65, 2901 (1990).

${ }^{47}$ A diagrammatic formulation for the shot noise in a quantum dot coupled to ferromagnetic leads can be seen in Ref. 56.

${ }^{48}$ F. M. Souza, J. C. Egues, and A. P. Jauho, Braz. J. Phys. 34, 565 (2004).

${ }^{49}$ W. Rudziński and J. Barnaś, Phys. Rev. B 64, 085318 (2001).

${ }^{50}$ Typical values of $\Gamma_{0}$ and $U$ can be found, for example, in $\mathrm{D}$. Goldhaber-Gordon, H. Shtrikman, D. Mahalu, D. AbuschMagder, U. Meirav, and M. A. Kastner, Nature (London) 391, 156 (1998); F. Simmel, R. H. Blick, J. P. Kotthaus, W. Wegscheider, and M. Bichler, Phys. Rev. Lett. 83, 804 (1999); A. Kogan, S. Amasha, D. Goldhaber-Gordon, G. Granger, M. A. Kastner, and H. Shtrikman, ibid. 93, 166602 (2004).

${ }^{51}$ These parameters correspond to the polarization of $\mathrm{Ni}$ and $\mathrm{Co}$, respectively. See R. Meservey and P. M. Tedrow, Phys. Rep. 238, 173 (1994).

${ }^{52}$ In terms of the conduction channels single occupancy means $\mu_{R}<\epsilon_{i}<\mu_{L}$ and $\epsilon_{i}+U>\mu_{L}, \mu_{R}$.

${ }^{53}$ See, for instance, Eq. (76) of Ref. 43.

${ }^{54}$ For nonmagnetic leads, closed expressions for the current and the shot noise in all the plateaus can be found in A. Thielmann, M. H. Hettler, J. König, and G. Schön, Phys. Rev. B 68, 115105 (2003).

${ }^{55}$ A suppression of the shot noise due to spin flip can also be seen in F. G. Brito and J. C. Egues, Braz. J. Phys. 32, 324 (2002); I. Djuric, B. Dong, and H. L. Cui, IEEE Trans. Nanotechnol. 4, 71 (2005).

${ }^{56}$ M. Braun, J. König, and J. Martinek, Phys. Rev. B 74, 075328 (2006).

${ }^{57}$ Comparing the current plateaus obtained from Eq. (16) and the ones derived in Ref. 54 we find a complete agreement between the results.

${ }^{58}$ H. B. Sun and G. J. Milburn, Superlattices Microstruct. 23, 883 (1998).

${ }^{59}$ Y. P. Li, A. Zaslavsky, D. C. Tsui, M. Santos, and M. Shayegan, Phys. Rev. B 41, 8388 (1990).

${ }^{60}$ J. H. Davies, J. C. Egues, and J. W. Wilkins, Phys. Rev. B 52 , 11259 (1995). In this work, shot noise in a Fabry-Perot type model with dephasing leads was analyzed and an enhancement of the Fano factor was found.

${ }^{61}$ See Chapter 12 of Ref. 41 for a discussion about this kind of approximation. 